\title{
Censoring-Based Relay Transmission for Achieving Energy Efficiency in Cognitive Radio Networks
}

\author{
Li Feng $\mathbb{D}^{1,2}{ }^{1,2}$ Jirong Sun, ${ }^{1}$ Hua Sun, ${ }^{1}$ and Shengju Tang ${ }^{1}$ \\ ${ }^{1}$ School of Engineering \& Technology, Sichuan Open University, Chengdu 610073, China \\ ${ }^{2}$ Educational Information Management and Information System Research Center of Open University of China (OUC), \\ Sichuan Open University, Chengdu 610073, China
}

Correspondence should be addressed to Li Feng; feng_fengli@163.com

Received 22 February 2020; Revised 31 July 2020; Accepted 6 August 2020; Published 8 September 2020

Academic Editor: Yujin Lim

Copyright (c) $2020 \mathrm{Li}$ Feng et al. This is an open access article distributed under the Creative Commons Attribution License, which permits unrestricted use, distribution, and reproduction in any medium, provided the original work is properly cited.

Energy efficiency (EE) is critical to achieve cooperative sensing and transmission in relay-assisted cognitive radio networks (CRNs) with limited battery capacity. This paper proposes an energy-efficient cooperative transmission strategy with combined censoring report and spatial diversity in cooperative process, namely, censoring-based relay transmission (CRT). Specifically, secondary relays (SRs) take part in cooperative sensing with differential censoring to reduce energy consumption, and the best SR assists secondary transmission to enhance communication quality in transmission stage and thus to improve secondary transmission EE. First, we derived generalized-form expressions for detection probability, reporting probability, sensing energy, and expected throughput for CRT. Second, we investigate a mean EE-oriented maximization nonconvex problem by joint optimizing sensing duration and power allocation for secondary users under secondary outage probability and sensing performance constraints. With the aid of Jensen's inequality, an efficient cross-iteration algorithm with low complexity is proposed to obtain the suboptimal solutions, which is developed by golden segmentation search method. Finally, extensive simulations are conducted to evaluate the performance of CRT. The results show significant improvements of SUs' EE compared with traditional noncooperation single cognitive transmission schemes, which demonstrate the benefits of our proposed cooperative strategy in conserving energy for secondary transmission.

\section{Introduction}

The energy consumption of communication systems has increased dramatically, making it an urgent issue in the information world to improve energy efficiency (EE) of mobile communications. The high energy consumption and exponential growth in wireless communication networks face serious challenges to the design of more EE and spectrum efficiency (SE) green communications that should deal with the scarcity of radio resources. A promising approach technique called cognitive radio networks (CRNs) is proposed as a key design to improve spectral efficiency of wireless communication. Especially, advancements in miniaturization of devices with higher computational capabilities and ultralow power communication technologies are driving forces for the ever growing deployment of embedded devices in our surroundings; how to achieve energy-efficient transmission becomes even more critical [1-3]. Cognitive relay networks have received a lot of interest to improve network performance by expanding the coverage and increasing SE [4]. It is well known that cooperative relaying promotes a diversity gain, improving spectrum efficiency and reducing the time used to deliver a message through distributed transmission and signal processing $[5,6]$. At the same time, the flexibility of cognitive radios is important to address the challenges and trade-offs between EE performance and practicality in $[2,7]$. As important means for improving SE, cognitive radio and cooperative transmission are also considered potential techniques to achieve green communication. By introducing cooperative transmission into CRNs, cooperative CRNs can not only improve SE but also overcome fading effect and thus attract much attention from researchers [4-10]. In this 
context, cooperation and cognition techniques can bring invaluable contributions to development of energy efficient networks.

In short, in this paper, our goal is to improve network EE while ensuring performance of secondary transmitting system. We propose an innovative best-relay-assisted transmission with censoring scheme to provide energy-efficient cooperative transmission in overlay CRNs. Our main contributions of this paper are summarized as follows:

(i) We propose a novel energy-efficient and best-relayassisted cooperative transmission scheme called CRT to reduce sensing energy overhead on sensing stage and enhance communication quality in transmission stage. CRT naturally integrates the censoring report, best-relay-assisted transmission, and time and power optimization in cooperative process, which can effectively improve secondary transmission $\mathrm{EE}$

(ii) On the basis of CRT scheme, we derive the generalized-form expressions for reporting probability, average energy, and average throughput for CRT. We formulate a mean EE-oriented maximization nonconvex problem by joint optimizing sensing duration and power allocation for secondary users under constraints of minimal sensing performance and secondary outage probability. Theoretical analysis demonstrates that the EE function of CRT scheme has a globally unique optimal solution. And then, an efficient cross-iteration algorithm was proposed to obtain the suboptimal solutions

(iii) Finally, we conduct extensive simulations to evaluate EE performance of CRT which is also compared with that of the noncooperative scheme. It is shown that CRT scheme can remarkably improve EE metrics of secondary transmission compared to the noncooperative case

The rest of this paper is structured as follows. Section 2 briefly describes any related work. The system model and transmission description is described in Section 3. In Section 4 , the proposed optimization problem is formulated, and sensing time and power allocation strategy and the algorithm are presented in Section 5. Simulation results are discussed in Section 6. Finally, the conclusions are drawn in Section 7.

\section{Related Work}

As discussed above, fast increasing popularity of powerful intelligent devices and vast penetration of mobile internet business cause explosive volume of wireless traffic, which in turn makes spectrum even more crowded and communication systems more energy-intensive. Therefore, EE has been considered one of the key features in the 5th-generation mobile communication and has attracted many research interests $[1,4,6]$. As important means for improving SE, cognitive radio and cooperative transmission are also considered potential techniques to achieve green communication. By introducing cooperative transmission into cognitive radio networks, cooperative CRNs can not only improve SE but also overcome fading effect and thus attract much attention from researchers.

Most recent research works on cooperative CRNs are mainly focused on improvement of access opportunities and transmission throughput; few of them are concerning on EE. However, it is necessary to study impacts on cooperative spectrum sensing (CSS) and cooperative transmission in cooperative CRNs when EE is concerned to meet lowcarbon communication requirement. Given the importance of this issue, recent studies have focused on energy-efficient secondary transmission in CRNs from different aspects and indicated that cognitive and cooperation technology can reduce network energy consumption in CRNs [8-18]. Collaborative sensing also known as CSS is an efficient spectrum sensing technique to improve the sensing accuracy in cognitive radio. However, it brings extra collaborative sensing overhead due to mutual exchange of large information among cognitive users. In CSS, the number of cooperative users, fusion rule, transmission power, and sensing time affects the energy efficiency (EE) of the CSS. The authors in $[8,9]$, investigate subcarrier transmission path selection and power allocation optimization problem with the goal of maximizing system EE in cognitive relaying links considering secondary QoS and total power budget, and a step-by-step strategy based on binary search-assisted ascent method is proposed. In [11], the authors proposed a cluster-based CSS strategy to maximize EE by optimizing the fusion rule, whereby the transmission power and sensing time were presented by the joint optimization problem. In [13], a spectrum sharing strategy is proposed via multiwinner auction with multiple bands to increase throughput of secondary system under constraint of minimum EE requirement for primary system. In [15], an EE-oriented "win-win" cognitive spectrum sharing scheme is proposed in heterogeneous cognitive radio networks. The authors in [5] investigated a trade-off between the secrecy throughput and EE in CRNs and proposed a cooperative spectrum sharing paradigm to improve both the secrecy throughput and the energy efficiency of primary users. Comprehensively considering sensing energy and detection performance, a differential reporting adaptive energy-efficient spectrum detection scheme was proposed [10], which can remarkably reduce sensing energy consumption with only slightly degrading detection performance. In [16], the authors modeled power allocation problem with the goal of maximizing the average EE of cognitive users in the fast-fading scene subject to a transmission interruption probability constraint and proposed an efficient power allocation strategy by using the fractional programming and Lagrange dual method. Similar to [16], the authors in [17] investigated EE maximization problem with sensing time and transmitting power as optimization variables. Different from noncooperative single cognitive transmission (NST) in [16-18] studied a weighted convex objective function of relay numbers with the consideration of global detection probability and bit error rate of cooperative transmission which is solved by applying numerical analysis to obtain the optimal relay numbers. 
Based on recent research in energy-efficient cooperative sensing and transmission in CRNs, the motivation of this paper is expressed as follows. Compared with single cognitive transmission $[16,17,19]$, CSS improves spectrum detection accuracy of a cognitive radio network. Clearly, more secondary relays (SRs) involving in spectrum detection require more energy consumption. On the other hand, when secondary system needs to meet higher rate requirements, longer transmission distance will inevitably consume a large amount of transmitting power and appear to be inefficient. In [14], a combined censoring scheme is proposed with the goal of minimizing the network energy consumption subject to a specific detection performance constraint. It is shown that such a system can attain high energy savings. Notice that the essence of censoring mechanism is by decreasing the number of decision reporting information to reduce energy consumption on CSS. In other words, the result is only transmitted, if it is deemed to be informative. Moreover, $[15,18]$ also studied the maximization of energy-efficient transmission problem considering the energy consumption of sensing and transmission two stages, but they did not utilize censoring report. Besides, we investigate secondary transmission performance characterized by constraint of cognitive transmission outage probability, which differs from previous works [15-18] without QoS requirements.

Note that, in this paper, $\mathbb{E}[\cdot]$ denotes the expectation operator; $\operatorname{Pr}\{\cdot\}$ represents the probability of a random variable; $\boldsymbol{Y}^{-1}(\cdot)$ denotes the inverse function of $Y(\cdot) ;|\Xi|$ denotes the number of set $\Xi ; \mathscr{F}_{S}$ represents the feasible region of programming; and $\cdot$ stands for the definition operator.

\section{System Model and Transmission Description}

3.1. System Model. Consider a cognitive radio system with the coexistence of a pair of primary users (PU) $U_{P 1}-U_{P 0}$, a pair of secondary users ST-SD, and $M$ secondary relay users SRs $\left(\Xi=\left\{\mathrm{SR}_{i} \mid i=1,2, \cdots, M\right\}\right)$ that are available to assist secondary sensing and transmission $[4,17,18]$. Primary spectrum owned by the primary network is divided into several narrowbands with fixed bandwidth. Like [2, 17], without loss of generality, we assume that the channels are modeled as independent Rayleigh fading, where $h_{L I}(L \in H, I \in H, L \neq I$, $\left.H=\mathrm{ST}, \mathrm{SD}, \mathrm{SR}_{i} \mid i=1,2, \cdots, M\right)$ denotes the fading coefficient. Besides, the additive white Gaussian noise (AWGN) received at $J=\{\mathrm{ST}, \mathrm{SRs}\}$ is denoted as $n_{J}$ which is with zero mean and power spectral density $\sigma_{n}^{2}$. From [16], when PU's signal is a complex PSK, we know that the false alarm probability and detection probability of energy detection (ED) with predefined detection threshold $\Delta_{T}$ for cognitive users are, respectively, written as $\phi_{f, k}(\tau)=Q\left(\kappa_{1} \sqrt{\tau f_{s}}\right)$ and $\phi_{d, k}(\tau)=$ $Q\left(-\kappa_{2} \sqrt{\tau f_{\mathrm{s}} \kappa_{3}}\right)$, where $f_{\mathrm{s}}$ is the sampling frequency, $\kappa_{1}=\Delta_{T} /$ $\sigma_{n}^{2}-1, \kappa_{2}=1+\gamma_{j}-\Delta_{T} / \sigma_{n}^{2}, \kappa_{3}=1 /\left(2 \gamma_{j}+1\right)$, and $\Lambda(z)=(1 /$ $\sqrt{2 \pi}) \int_{z}^{\infty} e^{-u^{2} / 2} d u$. It is noted that, in this paper, the AND rule is used for decision fusion criterion to improve secondary system transmission opportunity as well as satisfy detection threshold $Q_{d}^{\text {th }}$. For implementation simplicity, we assume that the received signal-to-noise ratio $\gamma_{j}$ at $\mathrm{SU}_{j}$ are the same value, i.e., $\gamma_{j}=\gamma$. We let $H_{0}$ (i.e., $\theta=0$ ) and $H_{1}$ (i.e., $\theta=1$ ) denote two standard hypotheses used in spectrum sensing corresponding to PU's absence and presence, respectively. According to $[13,15]$, the global detection probability and false alarm probability of CSS based on AND fusion rule can be, respectively, written as

$$
\begin{aligned}
Q_{f, \mathrm{AND}}(\tau) & =\prod_{k \in J} \phi_{f, k}(\tau)=\left(\Lambda\left(\kappa_{1} \sqrt{\tau f_{\mathrm{s}}}\right)\right)^{M+1}, \\
Q_{d, \mathrm{AND}}(\tau) & =\prod_{k \in J} \phi_{d, k}(\tau)=\left(\Lambda\left(-\kappa_{2} \sqrt{\tau f_{\mathrm{s}} \kappa_{3}}\right)\right)^{M+1} .
\end{aligned}
$$

Here, it should be pointed out that the secondary transmission opportunity is generally satisfied $Q_{f, \text { AND }}(\tau) \leq$ $\phi_{f, k}(\tau)<0.5$ in a cognitive radio network. So we can easily obtain $\kappa_{1}>0$ from $\phi_{f, k}(\tau)$. On the other hand, to avoid interference with PU's transmission, the global detection probability $Q_{d, \mathrm{AND}}(\tau)$ needs to be not lower than a predetermined detection probability value $Q_{d}^{\text {th }}$, and then, we can obtain the relation $\phi_{d, k}(\tau) \geq Q_{d, \mathrm{AND}}(\tau) \geq Q_{d}^{\text {th }} \geq 0.9>0.5$. Following, we have $\kappa_{2}>0$ by analyzing $\phi_{d, k}(\tau)$. To facilitate the upcoming analysis, the main parameters used are listed in Table 1 that are used in our analytical model. Based on the above discussion, we propose the CRT scheme to achieve energy-efficient transmission for overlay CRNs.

3.2. Scheme Description. As illustrated in Figure 1, CRT scheme adopts both censoring report and best-relayassisted strategies, which is completely different from the traditional noncooperative single cognitive transmission (NST). Each media access control (MAC) frame of SU is composed of sensing phase duration $\tau$ and transmission phase of duration $T_{S}-\tau$ [5-10]. We assume that SRs operate in a fixed time division multiple access (TDMA) manner which is commonly considered in existing studies $[16,17]$, where each MAC frame is composed of two consecutive durations called spectrum sensing phase and data transmission phase. CRT is periodically executed both in sensing and transmission phase at the beginning of each MAC frame. Besides, the following transmission phase is the same as conventional packet transmission processes [5-17]. The communication process of CRT scheme is described in detail below.

(i) Cooperative sensing with censoring: As can be seen from Figure 1, each sensing process with DCR consists of two essential parts: local decision phase and decision report phase occupying $\beta$ and $1-\beta$ fractions of $\tau$, respectively. The decision report phase is further split $(1-\beta) \tau$ into $M$ equal segments. Then, each decision report phase is organized as $M$ subslots $\left\{\tau_{0}, \tau_{1}, \cdots, \tau_{M}\right\}$ occupying $(1-\beta) / M$ fraction of $\tau$. In DCR, ST holds a $M$ bits buffer denoted as $\mathrm{ST}_{\text {buf }}=$ $[0,0, \cdots, 0]_{1 \times M}$ to store all $\mathrm{SR}_{i}$ 's latest reporting decision. Accordingly, $\mathrm{SR}_{i}$ needs a 1-bit buffer denoted as $\mathrm{SR}_{\text {buf }}^{k}=[0]_{1 \times 1}$ to save previous local decision result. $\mathrm{SR}_{1}$ makes its local decision $\widehat{\theta}_{R i}$ first and sends $x_{R_{i}}=1$ 
only when $\widehat{\theta}_{R i} \oplus \mathrm{SR}_{\mathrm{buf}}^{i}=1$. Hence, differential censoring report strategy in CRT can be formulated as

$$
\left\{\begin{array}{l}
\mathrm{SR}_{i} \operatorname{send} x_{\mathrm{SR}_{i}}=1, \quad \text { if } \widehat{\theta}_{R_{i}} \oplus \mathrm{SR}_{\mathrm{buf}}^{i}=1, \\
\mathrm{SR}_{i} \text { keep quiet, } \quad \text { if } \widehat{\theta}_{R_{i}} \oplus \mathrm{SR}_{\mathrm{buf}}^{i} \neq 1,
\end{array}\right.
$$

where $x_{R_{i}}=1$ means the current sensing result differs from its buffer content in $\tau_{1}$. Meanwhile, ST attempts to receive the indicator from $\mathrm{SR}_{1}$ in $\tau_{1}$. In this case, if $\mathrm{SR}_{1}$ 's indicator is received successfully, $\mathrm{ST}$ inverses the bit $\mathrm{SU}_{\text {buf }}(1)$ of buffer $\mathrm{SU}_{\text {buf }}$ and makes a decision by $\mathrm{SU}_{\text {buf }}(1)$. Otherwise, $\mathrm{ST}$ makes a decision by $\mathrm{SU}_{\text {buf }}(1)$ directly. Specific ideas of DCR strategy can be found in [5], which is omitted here due to space limitation

(ii) Cooperative transmission by best-relay assisted: From Figure 1, transmission phase $T_{S}-\tau$ in CRT is further divided into two equal subslots, which are, respectively, denoted as $T_{t 1}$ and $T_{t 2}$ satisfying $T_{t 1}=$ $T_{t 2}=0.5\left(T_{S}-\tau\right)$. In $T_{t 1}$, ST's data will broadcast to all secondary relay users $\mathrm{SRs}$ with transmit power $P_{s}$ in the idle band. Then, $\mathrm{SR}_{k *}$ with the largest endto-end SNR is selected as best-relay-assisted user in cooperative secondary relay set $\Xi=\left\{\mathrm{SR}_{1}, \cdots, \mathrm{SR}_{i}, \cdots\right.$, $\left.\mathrm{SR}_{M}\right\}$ and $|\Xi|=M$. The selection criterion of bestrelay SUs can be described as $\mathrm{SR}_{k *}=\max _{S R_{k} \in \Xi}(\mathrm{min}$ $\left.\left(h_{T R_{k}}, h_{R_{k} D}\right)\right)$. Motivated by the best-relay selection mechanism in [17], $\mathrm{SR}_{k *}$ forward received data with transmitting power $P_{R_{k}}$ to SD by decode and forward protocol in $T_{t 1}$. It is worth mentioning that cooperative sensing and cooperative transmission stages are all assisted by secondary relays $\Xi$ in CRT. In actuality, to save energy, ST can select an appropriate set of cooperative users based on instantaneous channel state [18]

\section{Secondary Sensing and Transmission Optimization}

4.1. Cooperative Sensing and Transmission Energy Overhead. Our main goal is to reduce the energy consumptions of sensing stage and satisfy sufficient accuracy. Next, we will examine the average energy of CSS, denoted as $\mathrm{En}_{\mathrm{CSS}}$. Without loss of generality, we assume that the circuit energy consumption of the secondary source users and the cooperative relay users are $P_{\mathrm{ci}}^{S}$ and $P_{\mathrm{ci}}^{R}$ and further assume that sensing and decision reporting consumption power for each cognitive user are denoted as $P_{\text {se }}$ and $P_{\mathrm{dr}}$ [10]. Note that we ignore the constant of circuit consumptions in analysis of $\mathrm{En}_{\mathrm{CSS}}$; i.e., $\mathrm{En}_{\mathrm{CSS}}$ consists of $P_{\mathrm{se}}$ and $P_{\mathrm{dr}}$ only, due to the circuit power consumptions for each cognitive user which is always equal $\tau\left(P_{\mathrm{ci}}^{S}+M P_{\mathrm{ci}}^{R}\right)$ both in traditional and CRT schemes. By definition, false alarm means that $\mathrm{PU}$ is detected under $H_{0}$ and detection indicates that $\mathrm{PU}$ is detected under $H_{1}$. Besides, miss detection is defined as that PU is undetected under $H_{1}$. Like $[5,12,15]$, as shown in Figure 2, whether PU is communicating or not can be modeled as a renewal process which
TABLE 1: System parameters.

\begin{tabular}{|c|c|}
\hline Symbol & Description \\
\hline$h_{L I}$ & Channel coefficient between the link $L \longrightarrow I$ \\
\hline$T_{S}$ & Time slot duration \\
\hline$\tau$ & Sensing phase duration of MAC frame \\
\hline$\gamma_{j}$ & Received signal-to-noise ratio at $S R_{j}$ \\
\hline$\beta$ & Sensing time allocation factor \\
\hline$M$ & Number of available secondary relays \\
\hline$P_{\mathrm{ci}}^{S}, P_{\mathrm{ci}}^{R}, P_{S}^{\max }$ & $\begin{array}{l}\text { Secondary source, relay circuit power } \\
\text { consumptions, and total transmission power }\end{array}$ \\
\hline$\phi_{f, k}, \phi_{d, k}$ & $\begin{array}{c}\text { False alarm probability and detection } \\
\text { probability of each SU }\end{array}$ \\
\hline$P_{S}, P_{R_{k}}$ & Transmitting power allocation on ST and $\mathrm{SR}_{k}$ \\
\hline$\Phi$ & Transition matrix of PU's state \\
\hline$Q_{f, \mathrm{AN} D}, Q_{d, \mathrm{AND}}$ & $\begin{array}{l}\text { Global detection probability and false } \\
\text { alarm probability of CSS }\end{array}$ \\
\hline$P_{\mathrm{se}}, P_{\mathrm{dr}}$ & $\begin{array}{l}\text { Sensing, decision reporting consumption } \\
\text { power for each SU }\end{array}$ \\
\hline $\mathrm{En}_{\mathrm{CSS}}$ & Average energy of CSS \\
\hline $\operatorname{Th}\left(\Theta_{E}\right)$ & Secondary transmitting average throughput \\
\hline $\operatorname{En}\left(\Theta_{E}\right)$ & $\begin{array}{l}\text { Secondary transmitting average energy } \\
\text { consumption }\end{array}$ \\
\hline$\eta_{\mathrm{EE}}$ & Mean energy efficiency \\
\hline$\phi_{B}, \phi_{I}$ & $\begin{array}{l}\text { Stationary probabilities for primary band } \\
\text { to be busy and idle }\end{array}$ \\
\hline$\phi_{S}^{\mathrm{CRT}}$ & Secondary outage probability \\
\hline$Q_{d}^{\text {th }}$ & $\begin{array}{l}\text { Minimum global detection probability of } \\
\text { secondary transmission }\end{array}$ \\
\hline$\xi_{S}^{\max }$ & $\begin{array}{c}\text { Maximum outage probability of secondary } \\
\text { transmission }\end{array}$ \\
\hline$\pi_{1}, \pi_{0}$ & Average busy and idle periods \\
\hline$\sigma_{n}^{2}$ & Noise power \\
\hline$R_{L I}(x)$ & Transmission data rate of the link $L \longrightarrow I$ \\
\hline
\end{tabular}

alternates between busy and idle states. The busy and idle periods can be assumed to be exponentially distributed which can be expressed as $f_{B}(t)=v e^{-v t}$ and $f_{I}(t)=\rho e^{-\rho t}$, where $v$ is the transition rate from busy to idle state and $\rho$ is the transition rate from idle to busy state.

Accordingly, the average busy and idle periods are $\pi_{1}=$ $1 / v$ and $\pi_{0}=1 / \rho$. Hence, the stationary probabilities for primary band to be busy and idle are given by $\phi_{B}=\pi_{1} /\left(\pi_{1}+\right.$ $\left.\pi_{0}\right)$ and $\phi_{I}=\pi_{0} /\left(\pi_{1}+\pi_{0}\right)$. From [16], the transition matrix $\Phi$ is given by

$$
\begin{aligned}
\Phi & =\left[\begin{array}{ll}
\phi_{I / I}(t) & \phi_{B / I}(t) \\
\phi_{I / B}(t) & \phi_{B / B}(t)
\end{array}\right] \\
& =\frac{1}{\pi_{1}+\pi_{0}}\left[\begin{array}{cc}
\pi_{0}+\pi_{1} \mathrm{e}^{-\left(\pi_{0}+\pi_{1}\right) t} & \pi_{1}-\pi_{1} \mathrm{e}^{-\left(\pi_{0}+\pi_{1}\right) t} \\
\pi_{0}-\pi_{0} \mathrm{e}^{-\left(\pi_{0}+\pi_{1}\right) t} & \pi_{1}+\pi_{0} \mathrm{e}^{-\left(\pi_{0}+\pi_{1}\right) t}
\end{array}\right] .
\end{aligned}
$$




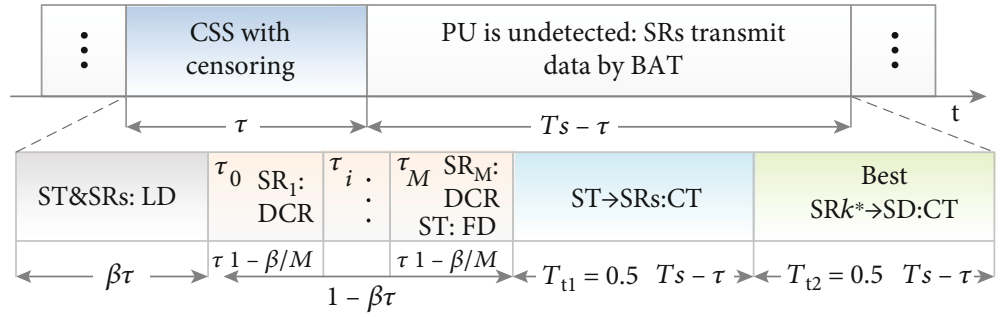

$\begin{array}{ll}\text { LD: local sensing } & \text { CSS: cooperative spectrum sensing } \\ \text { FD: fusion decision } & \text { DCR: differential censoring report } \\ \text { CT: cognitive transmission } & \text { BAT: best-relay assisted transmission }\end{array}$

FIgURE 1: Time slot structure of proposed cooperative sensing and transmission.

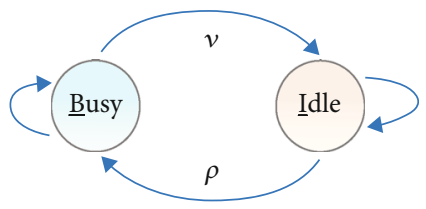

Figure 2: Continuous Markov transfer process of PU's channel.

For the purpose of comparison, the cooperative sensing energy consumption $\mathrm{En}_{\mathrm{CSS}}^{\text {tra }}$ of traditional scheme without censoring can be derived as

$$
\mathrm{En}_{\mathrm{CSS}}^{\mathrm{tra}}=\tau\left[\beta(M+1) P_{\mathrm{se}}+(1-\beta) P_{\mathrm{dr}} \phi_{\mathrm{dr}, R_{i}}^{\mathrm{Tra}}\right]
$$

where $\phi_{\mathrm{dr}, R_{i}}^{\mathrm{Tr}}=\phi_{B}\left(1-\phi_{d, R_{i}}\right)+\phi_{I}\left(1-\phi_{f, R_{i}}\right)$ is the average decision reporting probability by a traditional dedicated reporting channel [17]. It should be mentioned that, in order to fully guarantee primary transmission QoS, secondary transmission opportunity is generally satisfied $\phi_{f, J}<0.5<\phi_{d, J}(\tau)$ in a cognitive radio network. Analyzing (5), we can get

$$
\operatorname{En}_{\mathrm{CSS}}^{\left(\pi_{1}<\pi_{0}\right)}>\operatorname{En}_{\mathrm{CSS}}^{\left(\pi_{1}>\pi_{0}\right)}
$$

where $E n_{C S S}^{(\mathrm{I})}$ indicates the cooperative sensing energy consumption when condition (I) in $E n_{C S S}^{(\mathrm{I})}$ is satisfied.

Theorem 1. The average reporting probability by DCR is $\phi_{d r, R_{i}}^{C R T}=\left(o_{1}-o_{2}\right)\left(\pi_{0}+\pi_{1}\right)^{-2} e^{-\left(\pi_{0}+\pi_{1}\right) t}$, where

$$
\left\{\begin{array}{l}
o_{1}=\pi_{0}^{2} \phi_{f, R_{i}}+\pi_{0} \pi_{1}\left(1+\phi_{d, R_{i}}-\phi_{f, R_{i}}\right)+\pi_{1}^{2}\left(1-\phi_{d, R_{i}}\right) \\
o_{2}=2 \pi_{0} \pi_{1}\left(\phi_{d, R_{i}}-\phi_{f, R_{i}}\right) .
\end{array}\right.
$$

Proof. According to the initial status of $\mathrm{PU}$, sensing result, and PU's transition cases in time slot $\tau_{i}$, the SR decision censoring report scheme analysis for DCR can be, respectively, given as the following four possible scenarios as shown in

\begin{tabular}{|c|c|c|}
\hline $\begin{array}{l}\text { PU's initial } \\
\text { state }\end{array}$ & $\begin{array}{l}\text { PU's transition } \\
\text { cases in } \tau_{i}\end{array}$ & $\begin{array}{l}\text { SR }_{i} \text { 's censoring report } \\
\text { occurrence event }\end{array}$ \\
\hline \multirow{2}{*}{$H_{1}$} & Busy $\longrightarrow$ busy & $\begin{array}{l}\mathrm{PU} \text { is undetected under } H_{1} \\
\text { (misdetection) }\end{array}$ \\
\hline & Busy $\longrightarrow$ idle & $\begin{array}{l}\mathrm{PU} \text { is undetected under } H_{0} \\
\text { (correct detection) }\end{array}$ \\
\hline \multirow{2}{*}{$H_{0}$} & Idle $\longrightarrow$ idle & $\begin{array}{c}\mathrm{PU} \text { is detected under } H_{0} \\
\text { (false alarm) }\end{array}$ \\
\hline & Idle $\longrightarrow$ busy & $\begin{array}{c}\mathrm{PU} \text { is detected under } H_{1} \\
\text { (detection) }\end{array}$ \\
\hline
\end{tabular}
Table 2.
TABLE 2: Four cases of $\mathrm{SR}_{i}$ decision by differential censoring report.

From Table 2, decision censoring report probability $\phi_{\mathrm{dr}, R_{i}}^{\mathrm{CRT}}$ for $\mathrm{SR}_{i}$ in DCR can be calculated as

$$
\begin{aligned}
\phi_{\mathrm{dr}, R_{i}}^{\mathrm{CRT}}= & \sum_{n \in\{0,1\}} \sum_{X \in\{B, I\}} \phi\left(H_{n}\right) \operatorname{Pr}\left\{\widehat{\theta}_{R_{i}}=n \mid H_{n}\right\} \phi_{X \mid X}(t) \\
= & \phi\left(H_{1}\right) \phi_{B \mid B}(t) \operatorname{Pr}\left\{\widehat{\theta}_{R_{i}}=0 \mid H_{1}\right\} \\
& +\phi\left(H_{1}\right) \phi_{I \mid B}(t) \operatorname{Pr}\left\{\widehat{\theta}_{R_{i}}=0 \mid H_{0}\right\} \\
& +\phi\left(H_{0}\right) \phi_{I \mid I}(t) \operatorname{Pr}\left\{\widehat{\theta}_{R_{i}}=1 \mid H_{0}\right\} \\
& +\phi\left(H_{0}\right) \phi_{B \mid I}(t) \operatorname{Pr}\left\{\widehat{\theta}_{R_{i}}=1 \mid H_{1}\right\} \\
= & \phi_{B}\left[\phi_{B \mid B}(t)\left(1-\phi_{d, R_{i}}\right)+\phi_{I \mid B}(t)\left(1-\phi_{f, R_{i}}\right)\right] \\
& +\phi_{I}\left[\phi_{I \mid I}(t) \phi_{f, R_{i}}+\phi_{B \mid I}(t) \phi_{d, R_{i}}\right] \\
= & \phi_{B}+\phi_{d, R_{i}}\left(\phi_{I} \phi_{B \mid I}(t)-\phi_{B} \phi_{B \mid B}(t)\right) \\
& +\phi_{f, R_{i}}\left(\phi_{I} \phi_{I \mid I}(t)-\phi_{B} \phi_{I \mid B}(t)\right) .
\end{aligned}
$$

Therefore, substituting (4) into (8), after some algebra, $\phi_{\mathrm{dr}, R_{i}}^{\mathrm{CRT}}$ is proved.

In what follows, $\mathrm{En}_{\mathrm{CSS}}^{\mathrm{CRT}}$ represents the differential censoring sensing energy consumption in CRT, which can be calculated as

$$
\mathrm{En}_{\mathrm{CSS}}^{\mathrm{CRT}}=\tau\left[\beta(M+1) P_{s e}+(1-\beta) P_{\mathrm{dr}} \phi_{\mathrm{dr}, R_{i}}^{\mathrm{CRT}}(\tau(1-\beta) / M)\right]
$$


Next, we analyze the transmission rate and energy consumption for CRT scheme based on link status of $U_{P 1}-$ $U_{P 0}$ and global decision of $J$. For ease of analysis, let $\mathrm{Th}_{i}$ $(i=1,2,3,4)$ and $\mathrm{En}_{i}$ represent the secondary transmission rate and energy consumption in CRT. As shown in Figure 1, the throughput and power consumption in transmission stage can be discussed four scenarios as follows:

(1) Presence detection with probability $\phi_{B} Q_{d, \mathrm{AND}}(\tau)$ : primary link is busy while sensing result is correct. In this case, in order to avoid interference to the primary system, the secondary system is prohibited transmission. Consequently, transmission rate is zero, and energy consumption in this case can be calculated as $\operatorname{En}_{1}\left(\Theta_{E}\right)=\operatorname{En}_{\mathrm{CSS}}^{\mathrm{CRT}}+T_{S}\left(P_{\mathrm{ci}}^{S}+M P_{\mathrm{ci}}^{R}\right)$, where $\Theta_{E} \hat{=}\left[\tau, P_{s}, P_{R_{k}}\right]$

(2) Miss detection with probability $\phi_{B}\left(1-Q_{d, \mathrm{AND}}(\tau)\right)$ : primary link is busy while sensing result is incorrect. In this case, like $[12,13]$, PU's signal usually has a large interference to secondary transmission, which causes SU to be unable to correctly decode data, so secondary transmission rate is zero and secondary energy consumption can be expressed as $\operatorname{En}_{2}\left(\Theta_{E}\right)=$ $\mathrm{En}_{\mathrm{CSS}}^{\mathrm{CRT}}+T_{S}\left(P_{\mathrm{ci}}^{S}+M P_{\mathrm{ci}}^{R}\right)+0.5\left(T_{S}-\tau\right)\left(P_{S}+P_{R_{k}}\right)$

(3) False alarm with probability $\left(1-\phi_{B}\right) Q_{f, \text { AND }}(\tau)$ : primary link is idle while sensing result is incorrect. There is no transmission with energy consumption $\operatorname{En}_{3}\left(\Theta_{E}\right)=\operatorname{En}_{\mathrm{CSS}}+T_{S}\left(P_{\mathrm{ci}}^{S}+M P_{\mathrm{ci}}^{R}\right)$

(4) Absence detection with probability $\left(1-\phi_{B}\right)(1-$ $\left.Q_{f, \mathrm{AND}}(\tau)\right)$ : primary link is idle while sensing result is correct. Secondary transmission is successful with a rate of $0.5\left(T_{s}-\tau\right) \mathbb{E}[\widetilde{\mathrm{Th}}]$, where $\widetilde{\mathrm{Th}}=\min \left\{R_{T R_{k}}\left(P_{s}\right.\right.$ $\left.\left.h_{T R}\right) \cdot R_{R_{k}, D}\left(P_{R_{k}} h_{R_{k}, D}\right)\right\}$ and $R_{L I}(x)$ represents transmission data rate under bandwidth $W, R_{L I}(x) \triangleq W$ $\log _{2}\left(1+x / \sigma_{n}^{2}\right), \mathbb{E}(\cdot)$ indicates the expectation operation, and secondary energy consumption in this case is calculated as $\operatorname{En}_{4}\left(\Theta_{E}\right)=\operatorname{En}_{\mathrm{CSS}}+T_{S}\left(P_{\mathrm{ci}}^{S}+M P_{\mathrm{ci}}^{R}\right)+$ $0.5\left(T_{S}-\tau\right)\left(P_{S}+P_{R_{k}}\right)$

Based on above four cases, the expected energy consumption for CRT can be expressed as

$$
\begin{aligned}
\overline{\operatorname{En}}\left(\Theta_{E}\right)= & \sum_{i} \operatorname{En}_{i} \operatorname{Pr}\left\{\operatorname{En}=\operatorname{En}_{i}\right\} \\
= & \operatorname{En}_{\mathrm{CSS}}^{\mathrm{CRT}}+T_{S}\left(P_{\mathrm{ci}}^{S}+M P_{\mathrm{ci}}^{R}\right) \\
& +0.5\left(T_{S}-\tau\right)\left(P_{S}+P_{R_{k}}\right) \phi_{A}
\end{aligned}
$$

where $\phi_{A}=1-\phi_{B} Q_{d, \mathrm{AND}}(\tau)-\phi_{I} Q_{f, \mathrm{AND}}(\tau)$. As discussed above, $Q_{d, \text { AND }}(\tau)$. usually needs to reach a predetermined detection value $Q_{d, \mathrm{AND}}(\tau) \geq Q_{d}^{\mathrm{th}} \geq 0.9$, and then, $\phi_{B} Q_{d, \mathrm{AND}}(\tau)$ $\approx \phi_{B} Q_{d}^{\text {th }}$. So, we can approximate $\overline{\operatorname{En}}\left(\Theta_{E}\right)$ in [13] as follows:

$$
\operatorname{En}\left(\Theta_{E}\right)=\omega_{1}+0.5 \omega_{2} \phi_{A} \text {, }
$$

where $\omega_{1}=\operatorname{En}_{\mathrm{CSS}}+T_{S}\left(P_{\mathrm{ci}}^{S}+M P_{\mathrm{ci}}^{R}\right), \omega_{2}=-\left(T_{S}-\tau\right)\left(P_{S}+P_{R_{k}}\right)$, and $\phi_{A}=\phi_{I} Q_{f, \mathrm{AND}}(\tau)+\phi_{B} Q_{d}^{\text {th }}-1$.

Similarly, the expected secondary throughput in the CRT scheme can be derived as

$$
\begin{aligned}
\overline{\mathrm{Th}}\left(\Theta_{E}\right)= & 0.5\left(T_{S}-\tau\right) \phi_{I}\left(1-Q_{f, \mathrm{AND}}(\tau)\right) \mathbb{E} \\
& \cdot\left[\min \left\{R_{T R_{k}}\left(P_{S} h_{T R_{k}}\right), R_{R_{k} D}\left(P_{R_{k}} h_{R_{k} D}\right)\right\}\right] .
\end{aligned}
$$

As mentioned in Section 3.1, secondary link channel coefficient $h_{I J}$ obeys exponential distribution with mean $\lambda_{I J}$, so it is impossible to obtain the closed expression $\mathbb{E}[\widetilde{\mathrm{Th}}]$ in $\overline{\mathrm{Th}}\left(\Theta_{E}\right)$. However, we observed that $R_{L I}(x)$ is a concave function. Therefore, $\widetilde{T h}$ is still a concave function due to the concavity of $\min (\log )$ function. In order to obtain the closed expression $\overline{\mathrm{Th}}\left(\Theta_{E}\right)$, like [13], $\mathbb{E}[\widetilde{\mathrm{Th}}]$ is approximated by Jensen inequality relationship, and it can be described as

$$
\mathbb{E}[\widetilde{\mathrm{Th}}] \leq \widehat{\mathrm{Th}}=\min \left\{R_{T R_{k}}\left(P_{S} \lambda_{T R_{k}}\right), R_{R_{k}, D}\left(P_{R_{k}} \lambda_{R_{k}, D}\right)\right\}
$$

where $\widehat{\mathrm{Th}}=\min \left\{R_{T R_{k}}\left(P_{S} \mathbb{E}\left[h_{T R_{k}}\right]\right), R_{R_{k}, D}\left(P_{R_{k}} \mathbb{E}\left[h_{R_{k}, D}\right]\right)\right\}$. Furthermore, the expected secondary throughput in CRT can be expressed as

$$
\operatorname{Th}\left(\Theta_{E}\right)=0.5\left(T_{S}-\tau\right) \phi_{I}\left(1-Q_{f, \mathrm{AND}}(\tau)\right) \widehat{\mathrm{Th}}
$$

In summary, according to the primary link state and global decision probability, the secondary throughput and energy consumption expressions under a corresponding situation are discussed.

4.2. Energy-Efficient Resource Optimization Problem in CRT. Similar to $[12,13,17]$, mean EE is defined as a ratio between average throughput and average energy consumption, i.e., $\eta_{E E}^{\mathrm{CRT}}\left(\Theta_{E}\right) \triangleq T h\left(\Theta_{E}\right) / E n\left(\Theta_{E}\right)$. Resource allocation in the CRT maximizes the mean EE as an optimization goal while considering secondary transmission QoS requirements. It is noted that the outage probability is introduced here to characterize secondary transmission QoS. According to the Shannon channel coding theorem, when channel capacity is lower than transmission rate required, it is considered a communication interruption. Thus, the secondary outage probability $\phi_{S}^{\mathrm{CRT}}$ can be expressed as

$$
\begin{aligned}
\phi_{S}^{\mathrm{CRT}} & =\operatorname{Pr}\left\{0.5\left(1-\tau / T_{S}\right) \phi_{I}\left(1-Q_{f, \mathrm{AND}}(\tau)\right) \widehat{\mathrm{Th}}<\mathrm{Th}_{S}^{\mathrm{req}}\right\} \\
& =\phi_{I}\left(1-Q_{f, \mathrm{AND}}(\tau)\right) \operatorname{Pout}_{S}^{\mathrm{CRT}}\left(\tau, P_{S}, P_{R_{k}}\right)
\end{aligned}
$$

where $\operatorname{Pout}_{S}^{\mathrm{CRT}}\left(\tau, P_{S}, P_{R_{k}}\right) \triangleq \operatorname{Pr}\left\{0.5\left(1-\tau / T_{S}\right) \phi_{I} \widehat{\mathrm{Th}}<\mathrm{Th}_{S}^{\text {req }}\right\}$ and $\mathrm{Th}_{S}^{\text {req }}$ denotes the minimum secondary throughput. The EE maximization problem for CRT is denoted as P1, which is a resource allocation problem by joint optimizing sensing duration and power allocation for SU under constraints of sensing performance and minimal secondary outage probability. Mathematically, the optimization problem is modeled as 


$$
\begin{array}{ll}
\text { P1 }: & \max _{\Theta_{E}} \frac{0.5\left(T_{S}-\tau\right) \phi_{I}\left(1-Q_{f, \mathrm{AND}}(\tau)\right) \mathbb{E}\left[\min \left\{R_{T R_{k}}\left(P_{S} h_{T R_{k}}\right), R_{R_{k} D}\left(P_{R_{k}} h_{R_{k} D}\right)\right\}\right]}{\omega_{1}+0.5 \omega_{2} \phi_{A}} \\
\text { s.t. } \quad & C 1: Q_{d, \mathrm{AND}}(\tau) \geq Q_{d}^{\text {th }}, \\
& C 2: \phi_{B}\left(1-Q_{f, \mathrm{AND}}(\tau)\right) \operatorname{Pout}_{S}^{\mathrm{CRT}}\left(\tau, P_{S}, P_{R_{k}}\right) \leq \xi_{S}^{\max }, \\
& C 3: P_{S}+P_{R_{k}}+P_{c i}^{S}+M P_{c i}^{R}+\operatorname{En}_{\mathrm{CSS}}^{\mathrm{CRT}} \leq P_{S}^{\max }, \\
& C 4: \beta^{*}=\arg _{\beta \in[0,1]} \min _{\mathrm{En}}^{\mathrm{CRT}}(\tau), \\
& C 5: 0 \leq \tau \leq T_{S}, \quad P_{S} \geq 0, P_{R_{k}} \geq 0 .
\end{array}
$$

In $\mathrm{P} 1, Q_{d}^{\text {th }}, \xi_{S}^{\max }$, and $P_{S}^{\max }$, respectively, denote the minimum global detection probability, the maximum outage probability, and power consumption budgets of SUs. The constraint (16).C1 indicates that the global detection probability in CRT is not lower than a predefined detection threshold $Q_{d}^{\text {th }}$, thereby avoiding missed detection and causing interference to primary transmission. (16).C2 indicates that secondary outage probability is not greater than the present value $\xi_{S}^{\max }$, and thus, secondary transmission quality is guaranteed. In addition, constraint relationship (16).C3 indicates that secondary total energy consumption needs to be no greater than total power consumption budgets of SUs $P_{S}^{\max }$. It is noted that, like $[5,17]$, the saved sensing energy can be used for possible interweave transmission. Similar to the detection probability, $\mathrm{En}_{\mathrm{CSS}}^{\mathrm{CRT}}$ can be minimized by optimizing $\beta$ in CRT due to the fundamental tradeoff in sensing time allocation. So, it can be formulated as P2: $\min _{\beta} \operatorname{En}_{\mathrm{CSS}}^{\mathrm{CRT}}(\tau)$ s.t. $Q_{d, \mathrm{AND}}(\tau) \geq Q_{d}^{\text {th }}$. Actually, minimum sensing energy can be achieved by optimizing $\beta$ in CRT, which will be validated by the simulations in Section 6. In addition, both the objective function and constraints are very complicated, which makes solving P1 with a low complexity resource allocation strategy difficult. However, we observe that $\mathbb{E}[\widetilde{\mathrm{Th}}]$ and $\widetilde{\mathrm{Th}}$ in (13) have the same optimal sensing duration, which accounts for $\mathbb{E}[\widetilde{T h}]$ and $\widetilde{T h}$ that do not consist $\tau$. Based on this analysis, the original optimization problem P1 can be decoupled into P3 for optimizing sensing time $\tau$ and P4 for optimizing power allocation $\left\{P_{S}, P_{R_{k}}\right\}$ separately. Specifically, secondary EE is only determined by sensing time $\tau$ at a fixed power $\left\{P_{S}^{\circ}, P_{R_{k}}^{\circ}\right\}$. Consequently, P3 is described as follows:

$$
\mathrm{P} 3: \max _{\tau} \eta_{\alpha \mathrm{EE}}^{\mathrm{CRT}}(\tau)=\frac{\operatorname{Th}\left(\tau, P_{S}^{\circ}, P_{R_{k}}^{\circ}\right)}{\operatorname{En}\left(\tau, P_{S}^{\circ}, P_{R_{k}}^{\circ}\right)}=\frac{\operatorname{Th}_{\alpha}(\tau)}{\operatorname{En}_{\alpha}(\tau)}
$$$$
\text { s.t. } \quad C 1: \phi_{B}\left(1-Q_{f, \mathrm{AND}}(\tau)\right) \operatorname{Pout}_{S}^{\mathrm{CRT}}\left(\tau, P_{S}^{\circ}, P_{R_{k}}^{\circ}\right) \leq \xi_{S}^{\max } \text {, }
$$

$$
\text { (16).C1, (16).C4, (16).C5, }
$$

where $\operatorname{Th}_{\alpha}(\tau) \triangleq \operatorname{Th}\left(\tau, P_{S}^{\circ}, P_{R_{k}}^{\circ}\right)$ and $\operatorname{En}_{\alpha}(\tau) \triangleq \operatorname{En}\left(\tau, P_{S}^{\circ}, P_{R_{k}}^{\circ}\right)$ represent system throughput and energy consumption for a given transmission power $P_{S}^{\circ}, P_{R_{k}}^{\circ}$. Similarly, given a sensing time $\tau^{\circ}$, EE is only determined by SU's transmitting power in turn. Therefore, $\mathrm{P} 4$ can be expressed as

P4 : $\max _{P_{S}, P_{R_{k}}} \eta_{\beta \mathrm{EE}}^{\mathrm{CRT}}\left(P_{S}, P_{R_{k}}\right)=\frac{\operatorname{Th}\left(\tau^{\circ}, P_{S}, P_{R_{k}}\right)}{\operatorname{En}\left(\tau^{\circ}, P_{S}, P_{R_{k}}\right)}=\frac{\operatorname{Th}_{\beta}\left(P_{S}, P_{R_{k}}\right)}{\operatorname{En}_{\beta}\left(P_{S}, P_{R_{k}}\right)}$

s.t. $\quad C 1: \phi_{B}\left(1-Q_{f, \mathrm{AND}}\left(\tau^{\circ}\right)\right) \operatorname{Pout}_{S}^{\mathrm{CRT}}\left(\tau^{\circ}, P_{S}, P_{R_{k}}\right) \leq \xi_{S}^{\max }$,

$C 2: P_{S}+P_{R_{k}} \leq P_{S}^{\max }-P_{c i}^{S}-M P_{c i}^{R}-\operatorname{En}_{\mathrm{CSS}}^{\mathrm{CRT}}$ and (16).C5,

where $\operatorname{Th}_{b}(\tau) \triangleq \operatorname{Th}\left(\tau^{\circ}, P_{S}, P_{R_{k}}\right)$ and $\operatorname{En}_{b}(\tau) \triangleq \operatorname{En}\left(\tau^{\circ}, P_{S}, P_{R_{k}}\right)$ denote the secondary throughput and energy consumption in CRT scheme for a given sensing time $\tau^{\circ}$, respectively.

\section{Joint Sensing Time and Power Resource Allocation}

In this section, a joint sensing time and power resource allocation for CRT is discussed. The following lemmas are provided to solve $\mathrm{P} 3$ and $\mathrm{P} 4$. Hereafter, notations $[F]_{x}^{\prime} \triangleq$ $\partial F(x) / \partial x$ and $[F]_{x}^{\prime \prime} \triangleq \partial^{2} F(x) / \partial x^{2}$ are defined the first derivative and the second derivative of $F$ with respect to $x$ for ease of presentation.

Theorem 2. The objective function in P3 is a quasiconcave function with respect to $\tau$.

Proof. From (14), we can derive the first derivative of $\operatorname{Th}_{\alpha}(\tau)$ as follows:

$$
\left[\mathrm{Th}_{\alpha}\right]_{\tau}^{\prime}=0.5 \phi_{B}\left(Q_{f, \mathrm{AND}}(\tau)-1+\left(\tau-T_{S}\right)(M+1) \phi_{f}^{M}\left[\phi_{f}\right]_{f}^{\prime}\right) \widehat{\mathrm{Th}}
$$


where $\left[\phi_{f}\right]_{\tau}^{\prime}$ can be formulated as

$$
\left[\phi_{f}\right]_{\tau}^{\prime}=-\frac{\kappa_{1}}{2} \sqrt{\frac{f_{\mathrm{s}}}{2 \pi \tau}} e^{-(1 / 2) \tau f_{\mathrm{s}} \kappa_{1}^{2}},
$$

and the second-order derivative of $\operatorname{Th}_{\alpha}(\tau)$ can be further derived as

$$
\left[\mathrm{Th}_{\alpha}\right]_{\tau}^{\prime \prime}=\phi_{B}\left((M+1) \phi_{f}^{M}\left[\phi_{f}\right]_{\tau}^{\prime}+2\left(\tau-T_{S}\right)\left[Q_{f, \mathrm{AND}}\right]_{\tau}^{\prime}\right) \widehat{\mathrm{Th}}
$$

where $\left[Q_{f, \mathrm{AND}}\right]_{\tau}^{\prime \prime}$ and $\left[\phi_{f}\right]_{\tau}^{\prime \prime}$ are, respectively, written as

$$
\begin{gathered}
{\left[Q_{f, \mathrm{AND}}\right]_{\tau}^{\prime \prime}=(M+1) \phi_{f}^{M-1}\left[M\left(\left[\phi_{f}\right]_{\tau}^{\prime}\right)^{2}+\phi_{f}\left[\phi_{f}\right]_{\tau}^{\prime \prime}\right],} \\
{\left[\phi_{f}\right]_{\tau}^{\prime \prime}=\kappa_{1}\left(\frac{1}{4 \tau}+\frac{f_{\mathrm{s}} \kappa_{1}^{2}}{4}\right) \sqrt{\frac{f_{\mathrm{s}}}{2 \pi \tau}} e^{-(1 / 2) \tau f_{\mathrm{s}} \kappa_{1}^{2}} .}
\end{gathered}
$$

As discussion in Section 3.1, we have $\kappa_{1}>0, \kappa_{2}>0$, and $\sigma_{n}^{2}<\Delta_{T}<\sigma_{n}^{2}(1+\gamma)$. Analyzing (20) and (22), we have $\left[\phi_{f}\right]_{\tau}^{\prime}$ $<0$ and $\left[\phi_{f}\right]_{\tau}^{\prime \prime}>0$, which leads to $\left[Q_{f, \mathrm{AND}}\right]_{\tau}^{\prime}<0$ and $\left[Q_{f, \mathrm{AND}}\right]_{\tau}^{\prime \prime}>0$, then $\left[\mathrm{Th}_{\alpha}\right]_{\tau}^{\prime \prime}<0$, which means $\operatorname{Th}_{\alpha}(\tau)$ is concave function with respect to $\tau$. Obviously, $\omega_{1}$ in (11) is an affine function. It is not difficult to analyze that $\omega_{2}$ in (11) is a convex function and monotonically increasing on $\tau$, and $\phi_{A}$ is a convex function and monotonically decreasing on $\tau$. From [19], $\omega_{1} \omega_{2}$ is a convex function with respect to $\tau$. Consequently, we can obtain that $\operatorname{En}_{\alpha}(\tau)$ is convex function on $\tau$. From $\operatorname{Th}_{\alpha}(\tau)$ which is a concave function with respect to $\tau$, we can ready analyze that $\eta_{\alpha \mathrm{EE}}^{\mathrm{CRT}}(\tau)$ is a quasiconcave function with respect to $\tau$ by the definition of quasiconcave function in [20] (see Appendix A for detail).

Afterward, analyzing constraint (17).C3, we can obtain the mathematical characteristics described in Theorem 2.

Theorem 3. The constraint (17).C1 is equivalent to $\Omega(\tau)=$ $\ln \omega_{S, 1}^{C R T}+\left(\omega_{S, 2}^{C R T}-1\right) \omega_{S, 3}^{C R T} \leq 0$ between $\tau \in\left[0, T_{S}\right]$, and $\Omega(\tau)$ is concave with respect to $\tau ; \omega_{S, 1}^{C R T}, \omega_{S, 2}^{C R T}$, and $\omega_{S, 3}^{C R T}$ in $\Omega(\tau)$ are, respectively, expressed as

$$
\left\{\begin{array}{l}
\omega_{S, 1}^{C R T}=\left(1-\xi_{S}^{\max }\right)\left(\phi_{I}-Q_{f, A N D}(\tau) \phi_{I}\right)^{-1} \\
\omega_{S, 2}^{C R T}=2^{2 R_{S}^{r e q}} T_{S} /\left(W\left(T_{S}-\tau\right)\right) \\
\omega_{S, 3}^{C R T}=\sigma_{n}^{2} \frac{\left(\lambda_{T R_{k}} P_{S}+\lambda_{R_{k} D} P_{R_{k}}\right)}{\left(\lambda_{T R_{k}} \lambda_{R_{k} D} P_{S} P_{R_{k}}\right)}
\end{array}\right.
$$

\section{Proof. See Appendix B.}

According to Theorem 2 and Theorem 3, the optimal sensing duration time strategy is described in Theorem 4 specifically.
Theorem 4. The optimal sensing time allocation strategy for $P 3$ can be characterized as

$$
\tau^{*}=\max \left(\max \left(\tau^{\mathrm{det}}, \underline{\tau}\right), \min \left(\tau^{o p t}, \bar{\tau}\right)\right)
$$

where $\tau^{\operatorname{det}}=\left(f_{s} \kappa_{3} \kappa_{2}^{2}\right)^{-1}\left(Q^{-1}\left(\sqrt[M+1]{Q_{d}^{t h}}\right)\right)^{2}, \tau^{o p t}, \underline{\tau}$, and $\bar{\tau}$ are, respectively, written as

$$
\left\{\begin{array}{l}
\tau^{\text {opt }}=A^{-1}(0), \quad \tau \in\left[0, T_{S}\right] \\
\tau^{\text {Pout }}=B^{-1}(0), \quad \tau \in\left[0, T_{S}\right] \\
\underline{\tau}=\Omega^{-1}(0), \quad \tau \in\left[0, \tau^{\text {Pout }}\right] \\
\bar{\tau}=\Omega^{-1}(0), \quad \tau \in\left[0, \tau^{\text {Pout }}\right]
\end{array}\right.
$$

where $A(\tau)=\left[\eta_{\alpha E E}^{C R T} \Omega\right]_{\tau}^{\prime}, B(\tau)=[\Omega]_{\tau}^{\prime}$, and $\boldsymbol{\gamma}^{-1}(\cdot)$ denotes the inverse function of $Y(\cdot)$.

Proof. The first derivative of $\phi_{d}(\tau)$ and $Q_{d, \text { AND }}(\tau)$ on $\tau$ are, respectively, derived as

$$
\begin{aligned}
{\left[\phi_{d}\right]_{\tau}^{\prime} } & =\frac{\Lambda_{2}}{2} \sqrt{\frac{\Lambda_{3} f_{\mathrm{s}}}{2 \pi \tau}} \exp \left(-\frac{1}{2} \tau f_{\mathrm{s}} \Lambda_{3} \Lambda_{2}^{2}\right), \\
{\left[\mathrm{Q}_{f, \mathrm{AND}}\right]_{\tau}^{\prime}=} & (M+1) \phi_{d}^{M}\left[\phi_{d}\right]_{\tau}^{\prime} .
\end{aligned}
$$

From (27), we can get $\left[\phi_{d}\right]_{\tau}^{\prime}>0$ and $\sigma_{n}^{2}<\Delta_{T}<\sigma_{n}^{2}(1+\gamma)$. Examining (28) leads to $\left[Q_{f, \mathrm{AND}}\right]_{\tau}^{\prime}>0$, which indicates that $Q_{d, \mathrm{AND}}(\tau)$ is a monotonically increasing function on $\tau$, so constraint (16).C1 in P2 can be formulated as $\tau>\tau^{\text {det }}=$ $\left(f_{\mathrm{s}} \Lambda_{3} \Lambda_{2}^{2}\right)^{-1}\left(Q^{-1}\left(\sqrt[M+1]{Q_{d}^{\text {th }}}\right)\right)^{2}$. Additionally, $[\Omega]_{\tau}^{\prime \prime}>0$ in Theorem 3 shows that $B(\tau)$ is a monotonically increasing function on $\tau$. We have $B(\tau) \longrightarrow-\infty$ as $\tau \longrightarrow 0^{+}$and $B(\tau) \longrightarrow+\infty$ as $\tau \longrightarrow T_{S}^{-}$. In other words, $\Omega(\tau)$ monotonically decreases with respect to $\tau$ in $\left[0, \tau^{\text {Pout }}\right]$ and monotonically increases with respect to $\tau$ in $\left[\tau^{\text {Pout }}, T_{S}\right]$. Therefore, the necessary and sufficient condition of (17).C1 existing a feasible $\tau$ in $\left[0, T_{S}\right]$ is $\Omega\left(\tau^{\text {Pout }}\right) \leq 0$. Furthermore, one of the following cases may occur for $\Omega\left(\tau^{\text {Pout }}\right) \leq 0$ : (1) when $\tau \in\left[0, \tau^{\text {Pout }}\right], \Omega(\tau)$ monotonically decreases on $\tau \operatorname{in}\left[0, \tau^{\text {Pout }}\right]$, and $\lim \Omega(\tau)>0$, which indicates that there exists a unique $\underline{\tau}$ satisfying $\Omega(\underline{\tau})=0$; (2) when $\tau \in\left[\tau^{\text {Pout }}, T_{S}\right]$, similarly, we can get $\Omega(\tau)$ monotonically decreases on $\tau$ in $\left[\tau^{\text {Pout }}, T_{S}\right]$, and $\lim _{\tau \rightarrow T_{S}^{-}} \Omega(\tau)=+\infty$, which indicates that there exists a unique $\bar{\tau}$. Conversely, if $\Omega\left(\tau^{\text {Pout }}\right)>0$, there is no sensing time $\tau \in\left[0, T_{S}\right]$ to satisfy $\Omega(\tau) \leq 0$. Above all, the optimal sensing duration $\tau^{*}$ is obtained at the boundary point or the stagnation point $\tau^{\text {opt }}=A^{-1}(0)$. This depends on the zero point $\tau^{\text {Pout }}$ of the first derivative of (17).C1.

Similar to Theorem 2, $\eta_{\beta \mathrm{EE}}^{\mathrm{CRT}}\left(P_{S}, P_{R_{k}}\right)$ of $\mathrm{P} 4$ can be proved a quasiconcave function with respect to $\left\{P_{S}, P_{R_{k}}\right\}$. We will 
not go into details here to save space. Before solving P4, Theorem 5 is given.

Theorem 5. In the CRT scheme, when secondary EE achieves maximum, the power allocation in P4 should be satisfied $\lambda_{T R_{k}} P_{S}=\lambda_{R_{k} D} P_{R_{k}}$.

Proof. Prove by contradiction. According to the objective function in (16), secondary EE is not only related to ST $\longrightarrow$ SD but also limited byST $\longrightarrow \mathrm{SR}_{k}$. Suppose that secondary system EE achieves optimum, there exists a $\left\{\tilde{P}_{S}, \tilde{P}_{R_{k}}\right\}$, satisfying $R_{T R_{k}}\left(\tilde{P}_{S} \mathbb{E}\left[h_{T R_{k}}\right]\right) \neq R_{R_{k} D}\left(\tilde{P}_{R_{k}} \mathbb{E}\left[h_{R_{k} D}\right]\right)$. From (12), it can be seen that the secondary throughput $\operatorname{Th}_{b}\left(\tilde{P}_{S}, \tilde{P}_{R_{k}}\right)=0.5 \widehat{\mathrm{T} h}$ indicates that its rate depends on the smaller rate, which means one can reduce the transmit power of the larger rate hop in $R_{T R_{k}}\left(\tilde{P}_{S} \mathbb{E}\left[h_{T R_{k}}\right]\right)$ and $R_{R_{k} D}\left(\tilde{P}_{R_{k}} \mathbb{E}\left[h_{R_{k} D}\right]\right)$, leading to $R_{T R_{k}}\left(\tilde{P}_{S} \mathbb{E}\left[h_{T R_{k}}\right]\right)=R_{R_{k} D}\left(\tilde{P}_{R_{k}} \mathbb{E}\left[h_{R_{k} D}\right]\right)$. By the definition of EE function, i.e., $\eta_{E E}^{\mathrm{CRT}}\left(\Theta_{E}\right) \triangleq T h\left(\Theta_{E}\right) / E n\left(\Theta_{E}\right)$, reducing transmission power of the larger rate hop will increase system EE under the condition that the system rate is a constant. Obviously, the original hypothesis leads to contradiction. Therefore, the original proposition is true.

Theorem 5 shows that power allocation when secondary transmission EE achieves the maximum must satisfy the following relationship $P_{R_{k}}^{*}=P_{S}^{*} \lambda_{T R_{k}} \lambda_{R_{k} D}^{-1}$. So, the optimal power $\left\{P_{S}^{*}, P_{R_{k}}^{*}\right\}$ for $\mathrm{P} 4$ is described in Theorem 6 specifically.

Theorem 6. The optimal power allocation strategy for P3 is

$$
\left\{\begin{array}{l}
P_{S}^{*}=\max \left(\underline{P_{S}}, \min \left(K^{-1}(0), \overline{P_{S}}\right)\right), \\
P_{R_{k}}^{*}=P_{S}^{*} \lambda_{T R_{k}} \lambda_{R_{k} D}^{-1}, \\
\underline{P_{S}}=\left[2 \sigma_{n}^{2}\left(\omega_{S, 2}^{C R T}-1\right) /\left(\lambda_{T R_{k}} \ln \left(1 / \omega_{S, 1}^{C R T}\right)\right)\right]^{+}, \\
\overline{P_{S}}=\left(P_{S}^{\max }-E n_{C S S}^{C R T}-P_{c i}^{S}-M P_{c i}^{R}\right) /\left(1+\lambda_{T R_{k}} \lambda_{R_{k} D}^{-1}\right),
\end{array}\right.
$$

where $[x]^{+} \triangleq \max (x, 0)$ and $K\left(P_{S}\right)=\left[\eta_{\beta E E}^{C R T}\right]_{P_{s}}^{\prime \prime}, P_{S} \in\left[\underline{P_{S}}, \overline{P_{S}}\right]$.

Proof. According to (18).C2 in P4, the upper bound of $P_{S}$ is obtained as

$$
P_{S} \leq \overline{P_{S}}=\frac{P_{S}^{\max }-P_{c i}^{S}-M P_{c i}^{R}-E n_{C S S}^{C R T}}{1+\lambda_{T R_{k}} \lambda_{R_{k} D}^{-1}} .
$$

And from (18).C2 in P4, the lower bound of P4 can be derived as

$$
P_{S} \geq \underline{P_{S}}=\frac{2 \sigma_{n}^{2}\left(\omega_{S, 2}^{\mathrm{CRT}}-1\right)}{\lambda_{T R_{k}} \ln \left(1 / \omega_{S, 1}^{\mathrm{CRT}}\right)} .
$$

As discussed above, the optimal transmit power $P_{S}^{*}$ for $\mathrm{P} 4$ can be obtained either at both ends of the boundary $P_{S}$ or $\overline{P_{S}}$ taken at the stagnation point $P_{S}^{\text {opt }}=K^{-1}(0)$. This is depend- ing on the constraints (18).C1 and (18).C2 in P4. In addition, when secondary system EE achieves maximum, each rate for two hops should be equal. It indicates that the optimal power allocation satisfies relationship $P_{R_{k}}^{*} \lambda_{R_{k} D}=P_{S}^{*} \lambda_{T R_{k}}$. So Theorem 6 is proved.

Theorem 6 shows that the optimal power allocation problem can be obtained according to (18) for a given sensing time $\tau^{\circ}$. In what follows, aiming to maximize secondary EE, a joint resource allocation algorithm for sensing time $\tau$ and transmission power $\left\{P_{S}, P_{R_{k}}\right\}$ on ST and $\mathrm{SR}_{k *}$ is proposed. We know that closed-form expressions of false alarm and detection probabilities for CRT are available in (1) and (2), which only require average channel gains and thus can be estimated in prior. Consequently, to solve P2, we develop linear search methods by golden section search (GSS) method [20]. The steps of optimal sensing time allocation factor $\beta$ of P2 is expressed in Algorithm 1.

The pseudocode for CRT is presented in Algorithm 2. It is not difficult to observe that the key basis in Algorithm 2 is $\tau$ and $\left\{P_{S}, P_{R_{k}}\right\}$ alternating iterations according to Theorem 4 and Theorem 6 until $\left|e e^{[n+1]}-e e^{[n]}\right|<\xi_{e e}$ or does not satisfy the constraints of P3 and P4. For ease of analysis, we assume that sensing duration and transmission power after $n$ iteration are denoted as $\left\{\tau^{[n]}, P_{S}^{[n]}, P_{R_{k}}^{[n]}\right\}$. Theorem 6 guarantees that $P_{S}^{[n]}, P_{R_{k}}^{[n]}$ is available during the $n$th iteration, and substituting $\tau^{[n]}$ into (29) has $\eta_{\mathrm{EE}}^{\mathrm{CRT}}\left(\tau^{[n]}, P_{S}^{[n+1]}, P_{R_{k}}^{[n+1]}\right) \geq \eta_{\mathrm{EE}}^{\mathrm{CRT}}$ $\left(\tau^{[n]}, P_{S}^{[n]}, P_{R_{k}}^{[n]}\right)$. Similarly, $\tau^{[n+1]}$ can be calculated by Theorem 4, that is, substituting $P_{S}^{[n+1]}, P_{R_{k}}^{[n+1]}$ into (25) and (26) has $\eta_{\mathrm{EE}}^{\mathrm{CRT}}\left(\tau^{[n+1]}, P_{S}^{[n+1]}, P_{R_{k}}^{[n+1]}\right) \geq \eta_{\mathrm{EE}}^{\mathrm{CRT}}\left(\tau^{[n]}, P_{S}^{[n]}, P_{R_{k}}^{[n]}\right)$. In this way, the iterative process can continue until it is satisfied $\mid e e^{[n+1]}$ $-e e^{[n]} \mid<\xi_{e e}$. Therefore, in Algorithm 2, the following relationship exists:

$$
\eta_{\mathrm{EE}}^{\mathrm{CRT}}\left(\tau^{[n+1]}, P_{S}^{[n+1]}, P_{R_{k}}^{[n+1]}\right) \geq \eta_{\mathrm{EE}}^{\mathrm{CRT}}\left(\tau^{[n]}, P_{S}^{[n]}, P_{R_{k}}^{[n]}\right)
$$

Namely, $\eta_{\mathrm{EE}}^{\mathrm{CRT}}\left(\tau^{[n]}, P_{S}^{[n]}, P_{R_{k}}^{[n]}\right)(n=1,2, \cdots) \quad$ is increasing successively until convergence condition is satisfied.

Remark 7. Algorithm 2 for maximization secondary system EE of the CRT scheme has been proposed to obtain the optimal value $\Theta_{E}$ in (16). From (6), we observe that the system EE for the NST case with $M=0$, which means the CRT scheme can degrade the NST scheme. Consequently, we can readily obtain the $\mathrm{EE}$ optimal resource allocation for the NST scheme by Algorithm 2, which will be validated by the simulations in Section 6.

\section{Simulation Results and Discussions}

In this section, we will evaluate the performance of proposed CRT by some numerical and simulation results and then also compare it with the traditional overlay case as presented in Section 3.1. Without loss of generality, the ED introduced in $[16,17]$ is used for local sensing throughout these 


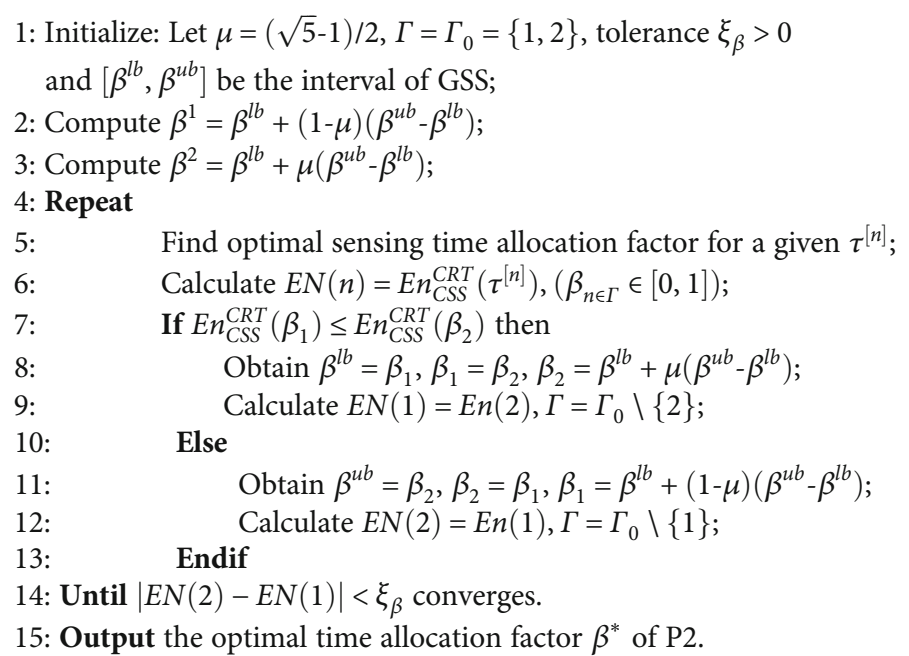

Algorithm 1: Obtain optimal sensing time allocation factor $\beta$ for CRT.

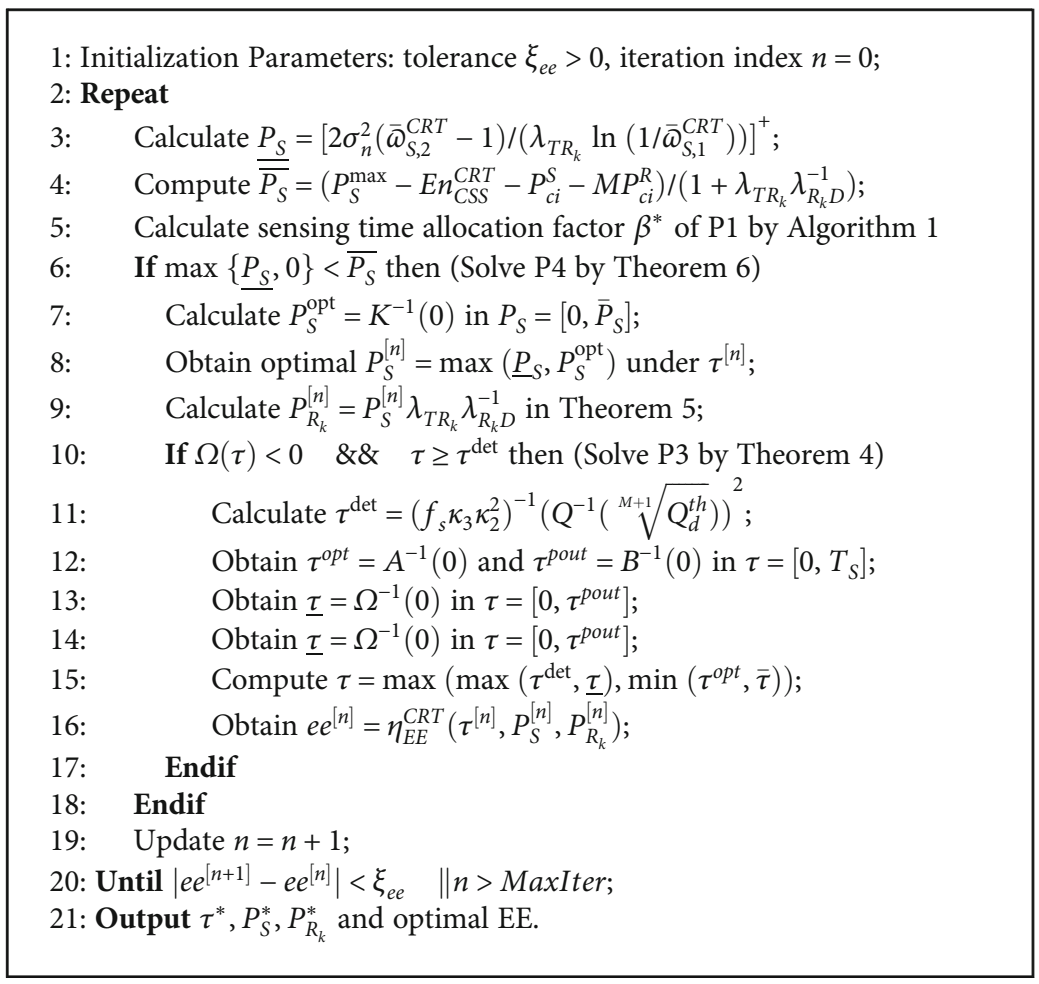

Algorithm 2: Joint sensing duration and transmission power allocation for CRT.

examples. Since we want to show the advantages of the proposed scheme, the choice of detector is not critical. Thus, the results obtained in this paper can be easily extended to other detector cases. Consider the scenario as follows. The secondary link distance between ST-SD is chosen as $1000 \mathrm{~m}$, and SRs are randomly distributed in the interval $[400,600] \mathrm{m}$. Throughout these simulation studies, unless otherwise noted, the simulation parameters are provided in Table 3.
First, we plot the global detection probability $Q_{d, \mathrm{AND}}$ versus the sensing time allocation factor $\beta$ for the traditional and CRT schemes in Figure 3. One can observe from Figure 3 that CRT experiences performance degradation compared with the traditional scheme due to the less local sensing time and decision reporting time. However, the minimum performance gap is rather small. Besides, such performance gap becomes smaller as $\gamma$ increases. Thus, under a reasonable detection probability requirement, CRT can not only 
TABLE 3: Simulation experiment parameter values.

\begin{tabular}{lccc}
\hline Parameters & Value & Parameters & Value \\
\hline$T_{S}$ & $0.1 \mathrm{~s}$ & $P_{S}^{\max }$ & $10 \mathrm{~W}$ \\
$\pi_{1}$ & $0.3 \mathrm{~s}$ & $f_{\mathrm{s}}$ & $10^{6} \mathrm{~Hz}$ \\
$\pi_{0}$ & $0.7 \mathrm{~s}$ & $R_{S}^{\text {req }}$ & $0.5 \mathrm{bps}$ \\
$P_{\mathrm{ci}}^{R}$ & $0.02 \mathrm{~W}$ & $P_{\mathrm{ci}}^{S}$ & $0.02 \mathrm{~W}$ \\
$P_{\mathrm{se}}$ & $0.02 \mathrm{~W}$ & $P_{\mathrm{dr}}$ & $0.04 \mathrm{~W}$ \\
$\sigma_{n}^{2}$ & $-64 \mathrm{dBm}$ & Path loss exponent & 3.5 \\
\hline
\end{tabular}

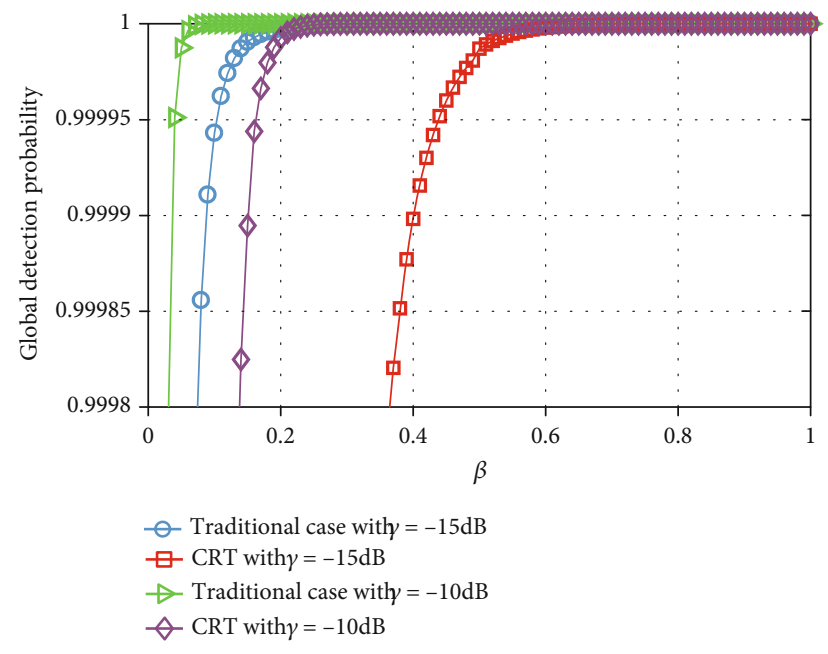

FIgURE 3: $Q_{d}$ versus $\beta$ for the traditional and proposed schemes.

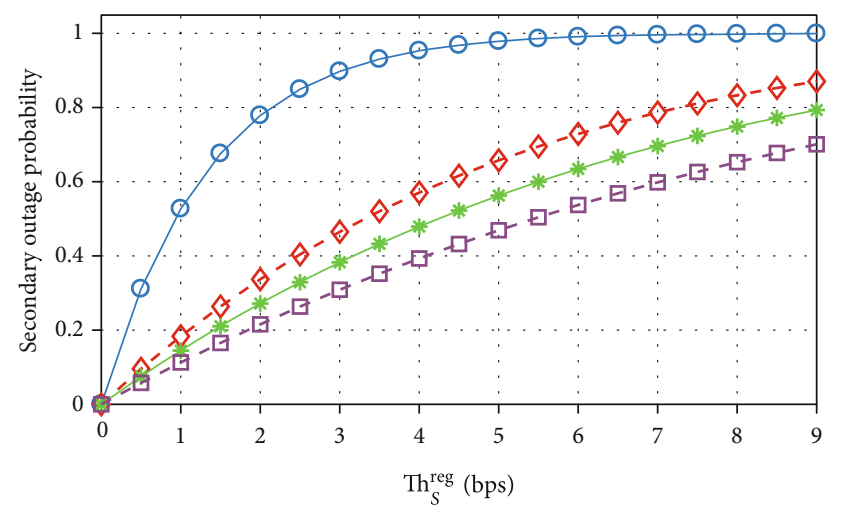

$$
\begin{array}{ll}
-M^{\prime} M=0, P_{S}^{\max }=1 \mathrm{~W} & -*-M=2, P_{S}^{\max }=1 \mathrm{~W} \\
-\diamond-M=1, P_{S}^{\max }=1 \mathrm{~W} & - \text { - } M=3, P_{S}^{\max }=1 \mathrm{~W}
\end{array}
$$

FIgURE 4: Secondary outage probability versus $\mathrm{Th}_{S}^{\text {req }}$.

maintain the sensing accuracy but also cut the sensing energy overhead. This will be validated later. Clearly, the global detection probability $Q_{d, \mathrm{AND}}$ increases with $\gamma_{P}$ growing. We also see that $\beta$ can be optimized to minimize the miss detection probability for CRT. Figure 4 shows the relationship between outage probability versus $\mathrm{Th}_{S}^{\text {req }}$ when different cooperative numbers are given. We observe that the best SR assists secondary communication to enhance QoS in the transmis-

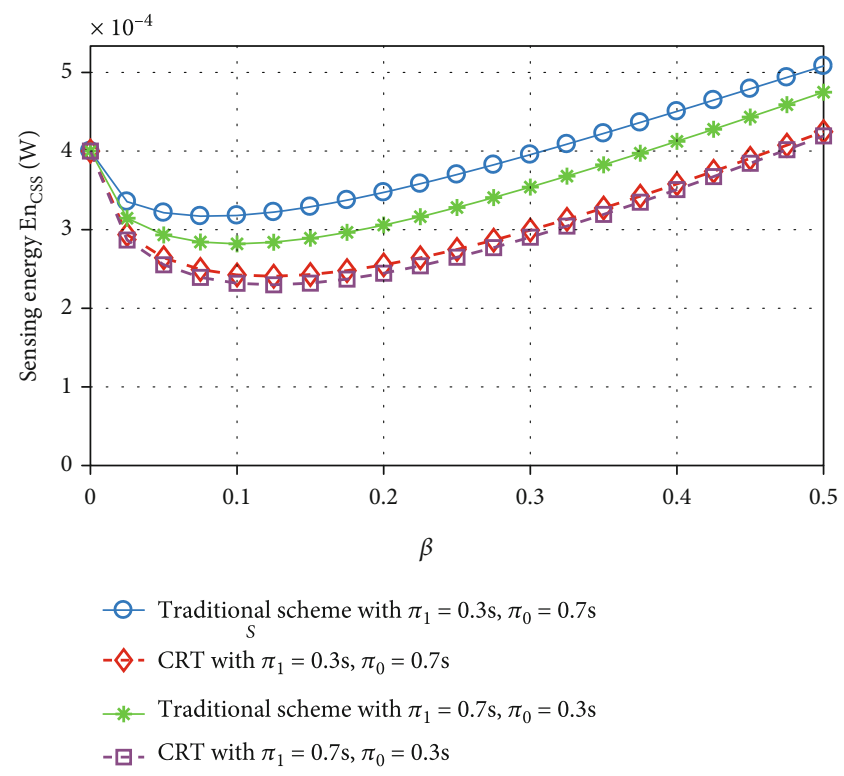

FIGURE 5: $\mathrm{En}_{\mathrm{CSS}}$ versus $\beta$ for the traditional and proposed schemes with $\gamma=-15 \mathrm{~dB}$.

sion stage. Furthermore, we also find that the secondary outage probability can be minimized by selecting a specific power allocation.

Second, we plot $\operatorname{En}_{\mathrm{CSS}}$ versus $\beta$ at $\gamma=-15 \mathrm{~dB}$ for the traditional and CRT schemes under PU's behavior. As shown in Figure 5 , it is observed that, under a given $\beta$, CRT remarkably reduce sensing energy consumption compared to the traditional case. It can be also noticed that $\mathrm{En}_{\mathrm{CSS}}$ of the traditional scheme with the $\pi_{1}=0.3 \mathrm{~s}$ and $\pi_{0}=0.7 \mathrm{~s}$ case is larger than the $\pi_{1}=0.8 \mathrm{~s}$ and $\pi_{0}=0.2 \mathrm{~s}$ case, which accounts for the fact that SRs send less decision reporting information by adopting censoring report than that without dedicated reporting channel strategy [17], and confirms the conclusions made in Section 4.1. As a further development, Figure 6 depicts the three-dimensional plot between $\operatorname{En}_{\mathrm{CSS}}$ and $\gamma$ and $\beta$. It is also revealed that $\beta$ can be optimized to minimize $\mathrm{En}_{\mathrm{CSS}}$ to achieve minimizing the miss detection probability and sensing energy overhead for CRT, however, e.g., $\mathrm{En}_{\min }=2.44 \times$ $10^{-4} \mathrm{~W}$ and optimal sensing time allocation factor $\beta=0.3$ under $\gamma=-9 \mathrm{~dB}$, which confirms the conclusions in Remark 7. Furthermore, we found that the optimal $\beta^{*}$ will decrease as $\gamma$ increases, for the reason that increasing $\gamma$ improves the local sensing reliability and thus, $\mathrm{PU}$ is detected to be more energy efficient.

Third, Figure 7 demonstrates the secondary EE and power consumption versus $\mathrm{Th}_{S}^{\text {req }}$. It is observed that, under a given secondary throughput requirement, CRT has remarkably improving EE compared to the traditional case in Figure 7. Moreover, the secondary transmission powers both in the traditional and CRT schemes increase with $\mathrm{Th}_{S}^{\text {req }}$ growing. Specifically, we can see clearly that the transmitting power is always increased with $\mathrm{Th}_{S}^{\text {req }}$ growing until achieving the maximum power budgets of SU. However, EE curves increase with the minimum rate requirement, while decrease slightly in larger $\mathrm{Th}_{S}^{\text {req }}$. This is due to the fact that 


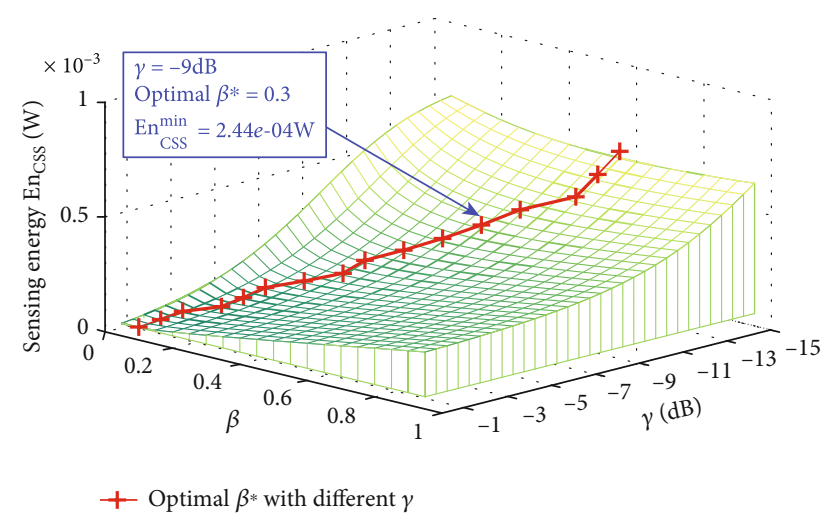

Figure 6: The three-dimensional plot between $\operatorname{En}_{\mathrm{CSS}}$ and $\gamma$ and $\beta$.

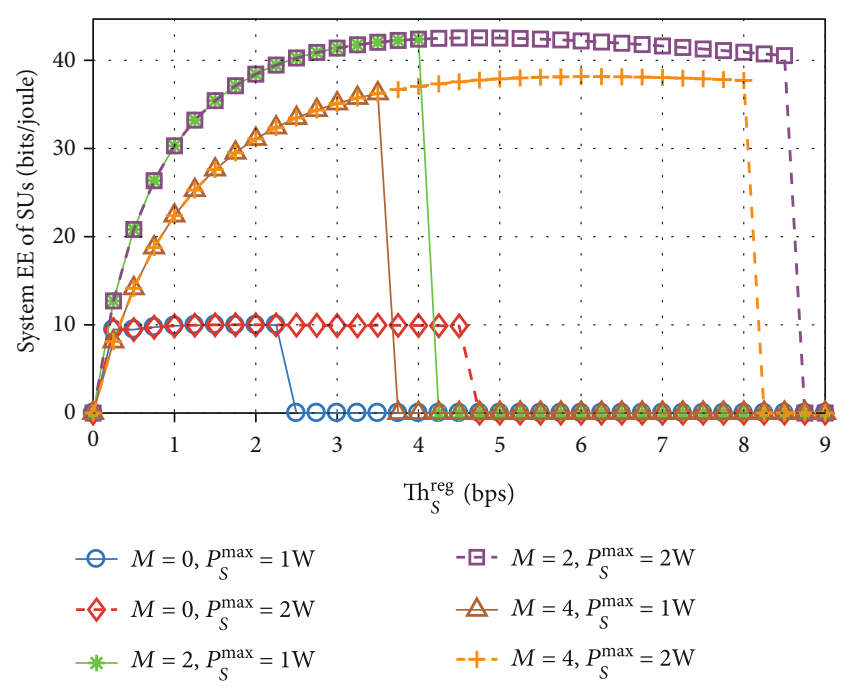

FIgURE 7: Secondary system EE versus $\mathrm{Th}_{S}^{\text {req }}$.

when the rate requirement is low, very low energy is needed to relay little data, which results in almost fixed throughput. On the other hand, when the rate requirement becomes high, more energy is needed to transmit data, which results in high expected throughput leading to a slight decrease in EE.

Finally, we depict the secondary EE versus $\gamma$ in Figure 8. It is observed that CRT has significantly improving EE compared to the traditional case. Clearly, system EE is almost fixed and relatively small in the low $\gamma$ region, which is due to the fact that secondary transmission is forbidden. However, as yincreases, system EE of CRT will increase, which accounts for the fact that the local sensing of SU will be sufficient to maintain detecting accuracy when $\gamma$ is high.

\section{Conclusion}

In this study, we propose an innovative energy-efficient cooperative transmission strategy, namely, censoring-based relay transmission (CRT). CRT naturally integrates differential censoring report, best-relay-assisted transmission technologies in cooperative process, which can effectively improve secondary transmission EE by reducing energy on sensing stage and enhancing communication quality in the

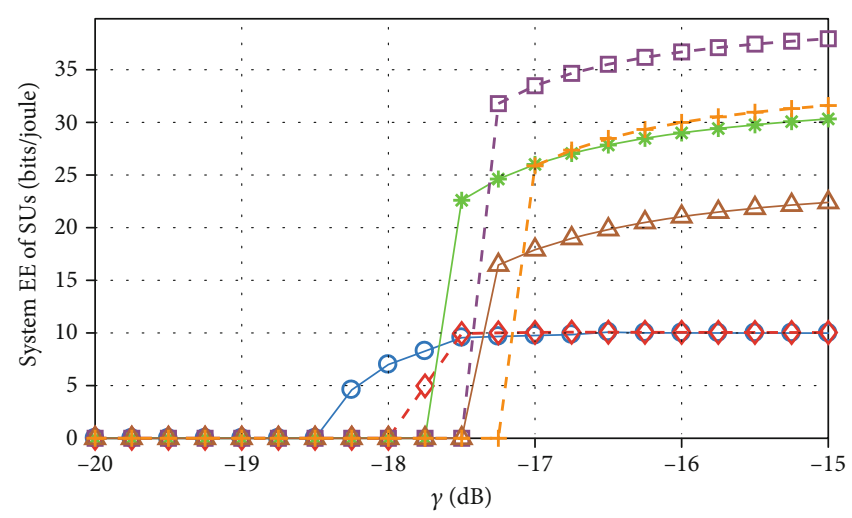

$$
\begin{array}{ll}
-M=0, R_{S}^{\mathrm{req}}=1 \mathrm{bps} & -\boxminus-M=2, R_{S}^{\mathrm{req}}=2 \mathrm{bps} \\
-\diamond-M=0, R_{S}^{\mathrm{req}}=2 \mathrm{bps} & -\triangle M=4, R_{S}^{\mathrm{req}}=1 \mathrm{bps} \\
-M=2, R_{S}^{\mathrm{req}}=1 \mathrm{bps} & -+-M=4, R_{S}^{\mathrm{req}}=2 \mathrm{bps}
\end{array}
$$

Figure 8: Secondary system EE versus $\gamma$.

transmission stage. On the basis of CRT, we derive generalized-form expressions for reporting probability, average energy, and expected throughput for CRT. We investigate a mean EE-oriented maximization nonconvex problem by joint optimizing sensing duration and power allocation for secondary users under constraints of minimal sensing performance and secondary outage probability. An efficient cross-iteration algorithm with low complexity is proposed to obtain the suboptimal solutions, which is developed by the golden segmentation search method. Finally, simulation results show that the proposed cooperative paradigm is an efficient approach to boost both SUs' throughput and EE of secondary transmission remarkably compared to traditional noncooperation cognitive transmission schemes in high QoS requirements.

\section{Appendix}

\section{A. Proof of Theorem 1}

Proof. Let $\left\{\tau_{1}, \tau_{2}\right\} \in \mathscr{F}_{\mathcal{\delta}}$, where $\mathscr{F}_{\mathcal{S}}$ represents the feasible region of P3 consisting the constraints (16).C1, (16).C5, and (17).C1, and further assuming that $0 \leq \vartheta \leq 1$ and $\eta_{\alpha \mathrm{EE}}^{\mathrm{CRT}}$ $\left(\tau_{1}\right)<\eta_{\alpha \mathrm{EE}}^{\mathrm{CRT}}\left(\tau_{2}\right)$, since $\mathrm{Th}_{\alpha}(\tau)$ is a concave function with respect to $\tau[10,20]$, we can express the following concave function:

$$
\begin{aligned}
\operatorname{Th}_{\alpha}\left(\vartheta \tau_{1}+(1-\vartheta) \tau_{2}\right) \\
>9 \operatorname{Th}_{\alpha}\left(\tau_{1}\right)+(1-\vartheta) \operatorname{Th}_{\alpha}\left(\tau_{2}\right) \\
>9 \operatorname{Th}_{\alpha}\left(\tau_{1}\right)+(1-\vartheta) \operatorname{En}_{\alpha}\left(\tau_{2}\right) \frac{\operatorname{Th}_{\alpha}\left(\tau_{1}\right)}{\operatorname{En}_{\alpha}\left(\tau_{1}\right)} \\
=\frac{\operatorname{Th}_{\alpha}\left(\tau_{1}\right)}{\operatorname{En}_{\alpha}\left(\tau_{1}\right)}\left(\vartheta \operatorname{En}_{\alpha}\left(\tau_{1}\right)+(1-\vartheta) \operatorname{En}_{\alpha}\left(\tau_{2}\right)\right) .
\end{aligned}
$$

In addition, $\operatorname{En}_{\alpha}(\tau)$ is a convex function on $\tau$, we have

$$
\operatorname{En}_{\alpha}\left(\vartheta \tau_{1}+(1-\vartheta) \tau_{2}\right)<\vartheta \operatorname{En}_{\alpha}\left(\tau_{1}\right)+(1-\vartheta) \operatorname{En}_{\alpha}\left(\tau_{2}\right)
$$


Substituting (A.2) into (A.1), the following relationship can be derived as

$$
\frac{\operatorname{Th}_{\alpha}\left(\vartheta \tau_{1}+(1-\vartheta) \tau_{2}\right)}{\operatorname{En}_{\alpha}\left(\vartheta \tau_{1}+(1-\vartheta) \tau_{2}\right)}>\frac{\operatorname{Th}_{\alpha}\left(\tau_{1}\right)}{\operatorname{En}_{\alpha}\left(\tau_{1}\right)}
$$

Analyzing (A.3), we can readily obtain that $\eta_{\alpha \mathrm{EE}}^{\mathrm{CRT}}(\tau)$ is a quasiconcave function with respect to $\tau$ by the definition of quasiconcave function in [20].

\section{B. Proof of Theorem 2}

Proof. This is due to $h_{T R_{k}}$ and $h_{R_{k} D}$ that obey exponential distribution with mean $\lambda_{T R_{k}}$ and $\lambda_{R_{k} D}$, respectively. According to (15), we can derive the following:

$$
\begin{aligned}
& \text { (16).C2 } \Rightarrow \phi_{I}\left(1-Q_{f, \mathrm{AND}}(\tau)\right) \operatorname{Pr}\left\{0.5\left(1-\tau / T_{S}\right) \widehat{\mathrm{Th}}<\mathrm{Th}_{S}^{\text {req }}\right\} \\
& \leq \xi_{S}^{\max } \Rightarrow \operatorname{Pr}\left\{0.5\left(1-\tau / T_{S}\right) \widehat{\mathrm{Th}}>\mathrm{Th}_{S}^{\text {req }}\right\} \\
& \geq \frac{1-\xi_{S}^{\max }}{\phi_{I}\left(1-Q_{f, \mathrm{AND}}(\tau)\right)} \Rightarrow \operatorname{Pr}\left\{0.5\left(1-\tau / T_{S}\right) \min \right. \\
& \left.\cdot\left\{R_{T R_{k}}\left(P_{S} h_{T R_{k}}\right), R_{R_{k} D}\left(P_{R_{k}} h_{R_{k} D}\right)\right\}>\mathrm{Th}_{S}^{\text {req }}\right\} \\
& \geq \omega_{S, 1}^{\mathrm{CRT}} \Rightarrow \operatorname{Pr}\left\{R_{T R_{k}}\left(P_{S}\right)>\frac{2 \mathrm{Th}_{S}^{\mathrm{req}} T_{S}}{T_{S}-\tau}\right\} \operatorname{Pr} \\
& \cdot\left\{R_{R_{k} D}\left(P_{R_{k}}\right)>\frac{2 \mathrm{Th}_{S}^{\mathrm{req}} T_{S}}{T_{S}-\tau}\right\} \geq \omega_{S, 1}^{\mathrm{CRT}} \\
& \Rightarrow \operatorname{Pr}\left\{h_{T R_{k}}>\frac{\left(\omega_{S, 2}^{\mathrm{CRT}}-1\right) \sigma_{n}^{2}}{P_{S}}\right\} \operatorname{Pr} \\
& \cdot\left\{h_{R_{k} D}>\frac{\left(\omega_{S, 2}^{\mathrm{CRT}}-1\right) \sigma_{n}^{2}}{P_{R_{k}}}\right\} \geq \omega_{S, 1}^{\mathrm{CRT}} \\
& \Rightarrow \exp \left(\frac{\left(1-\omega_{S, 2}^{\mathrm{CRT}}\right) \sigma_{n}^{2}}{\lambda_{T R_{k}} P_{S}}+\frac{\left(1-\omega_{S, 2}^{\mathrm{CRT}}\right) \sigma_{n}^{2}}{\lambda_{R_{k} D} P_{R_{k}}}\right) \\
& \geq \omega_{S, 1}^{\mathrm{CRT}} \Rightarrow \ln \omega_{S, 1}^{\mathrm{CRT}}+\left(\omega_{S, 2}^{\mathrm{CRT}}-1\right) \omega_{S, 3}^{\mathrm{CRT}} \leq 0 \text {. }
\end{aligned}
$$

So, the constraint (16).C2 is further equivalent to $\Omega(\tau)=\ln$ $\omega_{S, 1}^{\mathrm{CRT}}+\left(\omega_{S, 2}^{\mathrm{CRT}}-1\right) \omega_{S, 3}^{\mathrm{CRT}} \leq 0$, and the first derivation of $\Omega(\tau)$ can be formulated as

$$
[\Omega]_{\tau}^{\prime}=\left[Q_{f, \mathrm{AND}}\right]_{\tau}^{\prime} /\left(1-Q_{f, \mathrm{AND}}(\tau)\right)+\omega_{S, 3}^{\mathrm{CRT}}\left[\omega_{S, 2}^{\mathrm{CRT}}\right]_{\tau}^{\prime},
$$

where $\left[\omega_{S, 2}^{\mathrm{CRT}}\right]_{\tau}^{\prime}=2 \ln (2) T_{S} R_{S}^{\mathrm{req}} \omega_{S, 2}^{\mathrm{CRT}} W^{-1}\left(T_{S}-\tau\right)^{-2}$. The second derivative of $\Omega(\tau)$ is given by

$$
[\Omega]_{\tau}^{\prime \prime}=\omega_{S, 3}^{\mathrm{CRT}}\left[\omega_{S, 2}^{\mathrm{CRT}}\right]^{\prime \prime}{ }_{\tau}+\frac{\left[Q_{f, \mathrm{AND}}\right]_{\tau}^{\prime \prime}}{1-Q_{f, \mathrm{AND}}(\tau)}+\left(\frac{\left[Q_{f, \mathrm{AND}}\right]_{\tau}^{\prime}}{1-Q_{f, \mathrm{AND}}(\tau)}\right)^{2},
$$

where $\left[\omega_{S, 2}^{\mathrm{CRT}}\right]^{\prime \prime}{ }_{\tau}=2\left(1+\ln (2) T_{S} \mathrm{Th}_{S}^{\mathrm{req}} W^{-1}\left(T_{S}-\tau\right)^{-1}\right)\left[\omega_{S, 2}^{\mathrm{CRT}}\right]_{\tau}^{\prime}$. We can easy examine $\left[\omega_{S, 2}^{\mathrm{CRT}}\right]_{\tau}^{\prime \prime}>0$ and $[\Omega]^{\prime \prime}{ }_{\tau}>0$, which indicates $\Omega(\tau)$ is a concave function with respect to $\tau$.

\section{Data Availability}

The data used to support the findings of this study are included within this article.

\section{Conflicts of Interest}

All the authors hereby declare that there is no conflict of interest regarding the publication of this paper.

\section{Acknowledgments}

This work was mainly supported by the National Nature Science Foundation of China (No. 61801319), Key Research Project of OUC (No. G18F3319Z), Educational Reform Project of Sichuan Provincial Department of Education (No. JG2018-1240), Education Reform Project of Sichuan Open University (No. XMKYC2020004Z), and Outstanding Young Teacher Program of OUC.

\section{References}

[1] W. Yu, L. Musavian, and Q. Ni, "Tradeoff analysis and joint optimization of link-layer energy efficiency and effective capacity toward green communications," IEEE Transactions on Wireless Communications, vol. 15, no. 5, pp. 3339-3353, 2016.

[2] M. Liu, T. Song, J. Hu, J. Yang, and G. Gui, "Deep learninginspired message passing algorithm for efficient resource allocation in cognitive radio networks," IEEE Transactions on Vehicular Technology, vol. 68, no. 1, pp. 641-653, 2019.

[3] H. He and H. Jiang, "Deep learning based energy efficiency optimization for distributed cooperative spectrum sensing," IEEE Wireless Communications, vol. 26, no. 3, pp. 32-39, 2019.

[4] X. Liu, K. Zheng, L. Fu, X. Y. Liu, X. Wang, and G. Dai, "Energy efficiency of secure cognitive radio networks with cooperative spectrum sharing," IEEE Transactions on Mobile Computing, vol. 18, no. 2, pp. 305-318, 2019.

[5] L. Feng, Y. Kuang, B. Wu, Z. Dai, and Q. Yu, "Energy-efficient distributed spectrum sensing with combined censoring in cognitive radios," IEICE Transactions on Communications, vol. E99.B, no. 2, pp. 455-464, 2016.

[6] J. Gui, Z. Li, and Z. Zeng, "Improving energy-efficiency for resource allocation by relay-aided in-band D2D communications in C-RAN-based systems," IEEE Access, vol. 7, pp. 83588375, 2019.

[7] Z. Song, Q. Ni, K. Navaie, S. Hou, S. Wu, and X. Sun, "On the spectral-energy efficiency and rate fairness tradeoff in relayaided cooperative OFDMA systems," IEEE Transactions on Wireless Communications, vol. 15, no. 9, pp. 6342-6355, 2016.

[8] Y. Li, Z. Zhang, H. Wang, and Q. Yang, "SERS: social-aware energy-efficient relay selection in D2D communications," IEEE Transactions on Vehicular Technology, vol. 67, no. 6, pp. 5331-5345, 2018.

[9] L. Feng, Y. Kuang, and B. Wu, "Energy-efficient configuration of power resource for OFDM-based opportunistic regenerative 
relay links," Chinese Journal of Electronics, vol. 25, no. 2, pp. 341-347, 2016.

[10] L. Feng, Y. Kuang, X. Fu, and Z. Dai, “Energy-efficient network cooperation joint resource configuration in multi-RAT heterogeneous cognitive radio networks," Electronics Letters, vol. 52, no. 16, pp. 1414-1416, 2016.

[11] G. Sharma and R. Sharma, "Energy efficient collaborative spectrum sensing with clustering of secondary users in cognitive radio networks," IET Communications, vol. 13, no. 8, pp. 1101-1109, 2019.

[12] L. Wang, M. Sheng, X. Wang, Y. Zhang, and X. Ma, "Mean energy efficiency maximization in cognitive radio channels with PU outage constraint," IEEE Communications Letters, vol. 19, no. 2, pp. 287-290, 2015.

[13] L. Zhang, M. Xiao, G. Wu, S. Li, and Y. C. Liang, "Energy-efficient cognitive transmission with imperfect spectrum sensing," IEEE Journal on Selected Areas in Communications, vol. 34, no. 5, pp. 1320-1335, 2016.

[14] X. Xu, J. Bao, H. Cao, Y. D. Yao, and S. Hu, "Energy-efficiencybased optimal relay selection scheme with a BER constraint in cooperative cognitive radio networks," IEEE Transactions on Vehicular Technology, vol. 65, no. 1, pp. 191-203, 2016.

[15] S. Maleki and G. Leus, "Censored truncated sequential spectrum sensing for cognitive radio networks," IEEE Journal on Selected Areas in Communications, vol. 31, no. 3, pp. 364378, 2013.

[16] Y. C. Liang, K. C. Chen, Y. Li, P. Mahonen, and D. Niyato, "Guest editorial - advances in cognitive radio networking and communications (I)," IEEE Journal on Selected Areas in Communications, vol. 29, no. 2, pp. 273-275, 2011.

[17] Z. Dai, J. Liu, and K. Long, "Selective-reporting-based cooperative spectrum sensing strategies for cognitive radio networks," IEEE Transactions on Vehicular Technology, vol. 64, no. 7, pp. 3043-3055, 2015.

[18] J. M. Moualeu, T. M. N. Ngatched, W. Hamouda, and F. Takawira, "Energy-efficient cooperative spectrum sensing and transmission in multi-channel cognitive radio networks," in 2014 IEEE International Conference on Communications (ICC), pp. 4945-4950, Sydney, NSW, Australia, June 2014.

[19] J. Wu, G. Wang, and Y. R. Zheng, "Energy efficiency and spectral efficiency tradeoff in type-I ARQ systems," IEEE Journal on Selected Areas in Communications, vol. 32, no. 2, pp. 356366, 2014.

[20] S. Boyd and L. Vandenberghe, Convex Optimization, Cambridge University Press, New York, 2013. 